\title{
Exact one-loop false vacuum decay rate
}

\author{
Victor Guada $\odot^{*}$ and Miha Nemevšek $\oplus^{\dagger}$ \\ Jožef Stefan Institute, Jamova 39, 1000 Ljubljana
}

(Received 29 September 2020; accepted 19 November 2020; published 9 December 2020)

\begin{abstract}
We discuss an exact false vacuum decay rate at one loop for a real and complex scalar field in a quarticquartic potential with two tree-level minima. The bounce solution is used to compute the functional determinant from both fluctuations. We obtain the finite product of eigenvalues and remove translational zero modes. The orbital modes are regularized with the zeta function and we end up with a complete renormalized decay rate. We derive simple expansions in the thin and thick wall limits and determine their validity.
\end{abstract}

DOI: $10.1103 /$ PhysRevD.102.125017

\section{INTRODUCTION}

Tunneling phenomena are among the most fascinating physical processes. They initiate cosmological first order phase transitions, where an unstable ground state-a false vacuum $(\mathrm{FV})$ - transforms into an energetically favorable one. A bubble of true vacuum forms, expands quickly, collides with other bubbles and fills up the entire universe.

Theoretical studies of such transitions were initiated by Langer [1] and applied to field theory by [2] and notably by Coleman [3]. The decay rate was shown to be

$$
\Gamma \propto A e^{-\mathcal{S}_{0}}(1+\mathcal{O}(\hbar))
$$

where $\mathcal{S}_{0}$ is the saddle point Euclidean action and $A$ is a dimensionful prefactor.

Understanding bubble nucleation is crucial for several reasons. In the early universe, particles form a hot plasma, whose thermal effects can drive the transition [4] and dynamically generate the observed dominance of matter over antimatter, e.g., in electroweak baryogenesis [5-8]. Apart from creating baryons, colliding bubbles may produce observable gravitational wave signals [9-14] and primordial magnetic fields [15-19]. Current aLIGO [20] and aVIRGO [21] observatories are operating at frequencies that are mostly insensitive to $\mathrm{TeV}$ scale first order phase transitions, but upcoming detectors, such as LISA [22,23], DECIGO [24] and BBO [25,26], will have the ability to test such scenarios. Historically, vacuum stability played an important role in understanding the Higgs mass

\footnotetext{
*victor.guada@ijs.si

miha.nemevsek@ijs.si
}

Published by the American Physical Society under the terms of the Creative Commons Attribution 4.0 International license. Further distribution of this work must maintain attribution to the author(s) and the published article's title, journal citation, and DOI. Funded by SCOAP. bounds of the Standard Model (SM) from stability [27,28] and longevity [29]; see the recent works [30,31] and references therein.

The semiclassical picture in (1) is analogous to the path integral in quantum mechanics $[32,33]$, with the role of the classical trajectory replaced by the bounce. The bounce is a nontrivial unstable [34] configuration that extremizes the action and interpolates between the two minima of the potential. In [3], the bounce was found in the thin wall (TW) approximation, valid when the two vacua are nearly degenerate. It was also proven [35] that the dominant contribution to the rate is $O(4)$ symmetric, which simplifies the problem. Finding a closed form solution is in general impossible because one is dealing with a stiff nonlinear second order differential equation. Nonetheless, one can find exact solutions for specific potentials, including the Fubini-Lipatov instanton [36,37] and its generalization [38], linear [39,40], polygonal [41], logarithmic [42-44], pure quartic [45] and the quartic-quartic potential [46]. The situation becomes more involved with multiple scalar fields, where the bounce traverses a nontrivial path in field space. Nevertheless, the problem is understood and a number of tools [47-51] are available for a fast and stable evaluation of the action.

Phenomenologically, piecewise potentials have been used to estimate the bounce solutions, where closed-form results are not readily available. The triangular potential [39] was employed in estimating metastable supersymmetric minima, e.g., in $[52,53]$, in the context of dark energy [54] and gravitational wave production [55,56]. The quartic-quartic potential [46,57] and other combinations were studied in inflationary settings [46], where such glued bounce solutions are used $[58,59]$ as well. Other physically motivated examples [60] of false vacuum decay include the non-perturbative effects in 2 and 3 dimensions $[61,62]$.

The prefactor $A$ is a bit more challenging. It involves the calculation of a functional determinant [63], related to the operator that describes quantum fluctuations around the 
bounce. Not many closed form solutions exist, usually one estimates the dimensionful pre-factor $A$ by the inverse radius of the bounce solution. To get a more precise result, one can compute the rate numerically at zero [64-66] and finite temperatures $[67,68]$. For multifields, the bounce action is still $O(4)$ symmetric [69] and recently progress was made in numerical calculations for gauge theories [70].

An analytic estimate for the prefactor in the TW limit was found in [71] (see also [72-74]), while the issues with gauge and scale invariance of the unstable quartic in the SM were worked out recently $[30,31,75,76]$ and the FubiniLipatov was studied in [77]. The prefactor also cancels the renormalization scale-dependence of the Euclidean action and stabilizes the bubble nucleation rate, see [78] for a recent work on the uncertainties regarding gravitational wave production. However, to our knowledge, an exact solution for a potential with two separate tree level minima appears to be missing. We fill this gap here by finding such a new result for the decay rate of a quartic-quartic potential and its complexified version. We find a simple formula, where the energy scale of the FV factorizes and the rest of the prefactor depends only on the ratios of vevs and quartic couplings between the false and true vacuum. This setup can be considered as a benchmark for understanding the impact of finite one loop corrections, needed for a consistent evaluation of the total rate at one loop. In particular, one can easily derive the behavior in the thin and thick wall limits, thereby providing generic expectations for the class of potentials, which are approximately scale-invariant around the two minima.

There are a couple of subtleties that make the calculation of the functional determinant involved. The rate depends on an infinite product of eigenvalues of the fluctuation operator around the bounce, normalized to the FV ones. It turns out that it is not necessary to find the complete eigen-system with fixed boundary conditions. Instead, one can rely on the Gel'fand-Yaglom [79] theorem and solve a related differential equation with Cauchy boundary conditions. Evaluating it at the second boundary is equal to the product of eigenvalues. This is a considerably simpler procedure, even when it cannot be performed in closed form, which is typically the case.

The resulting spectrum contains a single negative eigenvalue [80] that describes the unstable direction of the expanding bubble. In addition, any symmetry (translational, scale or internal global invariance) of the bounce is reflected in the number of zero eigenvalues [81,82]. The quartic-quartic potential has no classical scale invariance, thus we only have to remove the four translational zero modes, which is done by a perturbative deformation of the homogeneous solution. This relates the dimensional prefactor to the energy scale in the theory and is proportional to the bounce action.

The final result is still infinite, as usual for quantities with a tree-level counterterm. We regularize it by subtracting the divergent asymptotic terms, expanded in a consistent power counting scheme. The asymptotic terms are added back in the UV, using the same power counting. This can be done with the effective action and Feynman diagrams or via the zeta function. We use the latter, where the zeta is defined via a contour integral [83] and its asymptotic form is calculated perturbatively in powers of the fluctuation potential [84]. We extend [84] to include the discontinuity of the quarticquartic fluctuation potential by going to higher orders in orbital eigenvalues to subtract all the infinities.

Finally, the UV terms need to be renormalized, either by computing the one loop counter-terms or by requiring the analyticity of the zeta function. Both correspond to the same renormalization scheme, i.e., to dimensional regularization in the $\overline{\mathrm{MS}}$ and give the same answer for the single quartic case.

We introduce the theoretical basis for the FV decay rate with quantum fluctuations in Sec. II. In Sec. III we define the quartic-quartic potential, set up the notation and review the bounce field configuration and the Euclidean action. Section Sec. IV deals with the functional determinants: the general formalism, exact expressions for the product of eigenvalues, removal of zeroes and the finite sum. In Sec. VA we review the zeta function formalism via the contour integral, then calculate the expansion around the FV in Sec. V B and get the finite and renormalized terms. Final result and thin/thick wall expansions are summarized in Sec. VI, with the complexified version in Sec. VI B. The outlook for further developments is discussed in Sec. VII and technical details are left to the Appendices A and B.

\section{FALSE VACUUM DECAY RATE}

The false vacuum decay rate was derived in [63] (see also [85]) and explained in more detail in Coleman's lectures $[86,87]$ and classic textbooks $[88,89]$. A recent rederivation $[90,91]$ used a more direct approach via the path integral formulation. The decay rate per space-time volume can be written as

$\frac{\Gamma}{\mathcal{V}}=\frac{\operatorname{Im} \int \mathcal{D} \varphi e^{-S[\bar{\varphi}]}}{\int \mathcal{D} \varphi e^{-S\left[\varphi_{\mathrm{FV}}\right]}}=\left(\frac{\mathcal{S}_{0}}{2 \pi}\right)^{2} e^{-\mathcal{S}_{0}} \operatorname{Im} \sqrt{\frac{\operatorname{det} \mathcal{O}_{\mathrm{FV}}}{\operatorname{det}^{\prime} \mathcal{O}}}(1+\mathcal{O}(\hbar))$

were $\int \mathcal{D} \varphi$ is the path integral over scalar field fluctuations and $S[\varphi]$ is the action functional in Euclidean space-time. The numerator is the path integral for real scalar fluctuations around the bounce field configuration, with an imaginary component, while the denominator is the FV normalization.

The Euclidean action $S[\varphi]$ is expanded around the bounce $\varphi=\bar{\varphi}+\psi$ to second order

$$
S[\varphi] \simeq S[\bar{\varphi}]+\frac{1}{2} \psi \mathcal{O} \psi+\ldots, \quad \mathcal{O}=\frac{\delta^{2} S}{\delta \varphi^{2}}[\bar{\varphi}] .
$$


The first derivative is zero because $\bar{\varphi}$ extremizes the action. The operator $\mathcal{O}$ is the fluctuation operator, defined as the second derivative of the action, evaluated on the bounce, while $\mathcal{O}_{\mathrm{FV}}$ is given in the $\mathrm{FV}$.

Expanding $\psi$ in a set of eigenfunctions $\psi_{\mathbf{n}}$ of the fluctuation operator $\mathcal{O} \psi_{\mathbf{n}}=\gamma_{\mathbf{n}} \psi_{\mathbf{n}}$, we perform the Gaussian integral in (2) and end up with the ratio of functional determinants [63]. The final step is to remove the translational zero modes by integrating over the collective coordinates [31,92-95], which produces the space-time volume factor $\mathcal{V}$ on the left hand side of (2) and the $\sqrt{\mathcal{S}_{0} /(2 \pi)}$ for every dimension of space-time. We thus end up with (2), where the prime in $\operatorname{det}^{\prime}$ corresponds to the four removed eigenvalues, each with dimension of mass ${ }^{2}$, which gives the correct dimension of the decay rate.

\section{THE BOUNCE SOLUTION}

The leading semiclassical approximation, i.e., the $\mathcal{S}_{0}$ coefficient in (1) and (2), is given by the bounce field configuration $\bar{\varphi}(\rho)$. The manifest dependence on $\rho^{2}=$ $t^{2}+\sum x_{i}^{2}$ takes care of Euclidean spherical symmetry [35]. Extremizing the action $S[\varphi]$ leads to the bounce equation

$$
\ddot{\varphi}+\frac{3}{\rho} \dot{\varphi}=V^{\prime}(\varphi)
$$

where $V^{\prime}=\mathrm{d} V / \mathrm{d} \varphi$ and with the boundary conditions $\dot{\bar{\varphi}}(0, \infty)=0$, where the bounce interpolates between the true $\bar{\varphi}(0)=\varphi_{0} \simeq \varphi_{\mathrm{TV}}$ and the false vacuum $\bar{\varphi}(\infty)=\varphi_{\mathrm{FV}}$.

Let us consider the exact bounce solution for the quarticquartic potential

$$
\begin{aligned}
V= & \frac{1}{4}\left(\lambda_{2} v_{2}^{4}-\lambda_{1} v_{1}^{4}+\lambda_{1}\left(\varphi+v_{1}\right)^{4}\right) H(-\varphi) \\
& +\frac{\lambda_{2}}{4}\left(\varphi-v_{2}\right)^{4} H(\varphi) .
\end{aligned}
$$

where $H$ is the step function. The two segments are joined at $\varphi=0$ into a continuous $V$ with the minima located at $-v_{1}$ and $v_{2}$. We assume that $v_{2}>0$ is the FV with $V\left(v_{2}\right)=0$ to simplify the bounce calculation. Furthermore, for $v_{1}>0$ to be the true vacuum (TV), we need $\lambda_{1,2}>0$ and require $\lambda_{1} v_{1}^{4}>\lambda_{2} v_{2}^{4}$ such that $V\left(v_{1}\right)<0$.

The derivative of the potential that enters in (4) is

$$
V^{\prime}=\lambda_{1}\left(\varphi+v_{1}\right)^{3} H(-\varphi)+\lambda_{2}\left(\varphi-v_{2}\right)^{3} H(\varphi) .
$$

The Dirac deltas, coming from the derivative of the step function in (5), vanish due to the continuity of $V$ at $\varphi=0$. Such potential was studied in [46] and admits an exact solution consisting of two pieces, glued together at $\rho=R_{T}$ $\bar{\varphi}=\sum_{s=1}^{2}\left((-1)^{s} v_{s}+\sqrt{\frac{8}{\lambda_{s}}} \frac{R_{s}}{R_{s}^{2}-\rho^{2}}\right) H\left((-1)^{s}\left(\rho-R_{T}\right)\right)$.

For later convenience, we define two dimensionless quantities $x$ and $y$ from $v_{1}=x v_{2}=x v$, and $\lambda_{1}=y \lambda_{2}=y \lambda$. Demanding the potential to be convex, ${ }^{1}$ implies $x>0, y>0$ and $x^{4} y>1$. Near the equality $x^{4} y \simeq 1$, we approach the TW limit, where the minima are degenerate and the rate vanishes. The bounce parameters $R_{1,2, T}$ are obtained by matching the solution to $\varphi=0$, and requiring $\bar{\varphi}$ to be continuous and differentiable at $\rho=R_{T}$. The resulting Euclidean radii are

$$
R_{1,2, T}=\frac{2}{v} \sqrt{\frac{2}{\lambda}} \frac{1+x}{x^{4} y-1}\left\{x^{2} \sqrt{y}, 1, \sqrt{\frac{x+x^{4} y}{1+x}}\right\} .
$$

Their size is set by $1 / v$, as expected on dimensional grounds since $v$ is the relevant mass scale. Moreover, the radii are positive and diverge in the TW limit $x^{4} y \rightarrow 1^{+}$, where the tunneling rate goes to zero. The resulting bounce action is

$$
\begin{aligned}
\mathcal{S}_{0} & =2 \pi^{2} \int_{0}^{\infty} \mathrm{d} \rho \rho^{3}\left(\frac{1}{2} \dot{\bar{\varphi}}^{2}+V(\bar{\varphi})\right) \\
& =\left(\frac{8 \pi^{2}}{3 \lambda}\right) \frac{1+y+x^{3} y\left(4+x y\left(-3+6 x^{2}+(3+4 x) x^{4} y\right)\right)}{y\left(x^{4} y-1\right)^{3}} .
\end{aligned}
$$

The factor of $8 \pi^{2} /(3 \lambda)$ is the well-known single quartic result, which gets multiplied by a function that diverges when $x^{4} y \rightarrow 1$ and thus $\Gamma \propto e^{-\mathcal{S}_{0}} \rightarrow 0$. With the bounce at hand, we can proceed to make sense of the quantum fluctuations.

\section{FUNCTIONAL DETERMINANTS}

As discussed in Sec. II, we are interested in calculating the spectra of eigenvalues of the fluctuation operator $\mathcal{O}$, appearing in (3). To this end, we employ the radial decomposition in four dimensions and get the product of eigenvalues for a fixed orbital momentum mode $l$, by use of the Gel'fand-Yaglom theorem [79].

\section{A. Radial mode separation and exact product of eigenvalues}

We would like to find the product of eigenvalues $\gamma_{\mathbf{n}}$, associated to $\mathcal{O}$

$$
\mathcal{O}=-\square+V^{\prime \prime}(\bar{\varphi}), \quad \mathcal{O} \psi_{\mathbf{n}}=\gamma_{\mathbf{n}} \psi_{\mathbf{n}},
$$

where $\square$ is the Laplace operator in flat 4D Euclidean spacetime. Here, $\mathbf{n}$ is a collective index for the relevant quantum

\footnotetext{
${ }^{1}$ One can also consider negative $\lambda$ and reproduce the SM instability, as we will see below.
} 
numbers that come about when the boundary conditions $\psi_{\mathbf{n}}(0)=\psi_{\mathbf{n}}(\infty)=0$ are imposed. The fluctuation potential follows from (6)

$$
\begin{aligned}
V^{\prime \prime}= & 3\left(\lambda_{1}\left(\varphi+v_{1}\right)^{2} H(-\varphi)+\lambda_{2}\left(\varphi-v_{2}\right)^{2} H(\varphi)\right) \\
& -\left(\lambda_{1} v_{1}^{3}+\lambda_{2} v_{2}^{3}\right) \delta(\varphi),
\end{aligned}
$$

and contains a delta function due to the discontinuity of $V^{\prime}$ at the origin. The $V^{\prime \prime}(\bar{\varphi}(\rho))$ is 4D symmetric, therefore we can separate the radial and orbital part of $\psi_{\mathbf{n}}$, where the latter is described by hyperspherical harmonics. These are eigenfunctions of the total orbital momentum operator with orbital quantum numbers $l=0, \ldots, \infty$ that are $(l+1)^{2}$ fold degenerate [33]. According to the Gel'fand-Yaglom theorem [79], we have to find the zero eigenmode of the fluctuation operator

$\mathcal{O}_{l} \psi_{l}=-\ddot{\psi}_{l}-\frac{3}{\rho} \dot{\psi}_{l}+\frac{l(l+2)}{\rho^{2}} \psi_{l}+V^{\prime \prime}(\bar{\varphi}) \psi_{l}=0$,

and evaluate $\psi_{l}$ at the boundary when $\rho \rightarrow \infty$. This gives the $\log$ of the ratio of determinants

$\ln \left(\frac{\operatorname{det} \mathcal{O}}{\operatorname{det} \mathcal{O}^{\mathrm{FV}}}\right)=\sum_{l=0}^{\infty}(l+1)^{2} \ln \mathcal{R}_{l}(\infty), \quad \mathcal{R}_{l} \equiv \frac{\psi_{l}}{\psi_{l}^{\mathrm{FV}}}$.

Let us see how the fluctuations behave. In the FV, we have $V_{\mathrm{FV}}^{\prime \prime}=0$ and the solution of (13) is $\psi_{l}^{\mathrm{FV}}=\rho^{l}$. We dropped the part that diverges at $\rho=0$ and assigned the arbitrary multiplication constant to 1 . The general solution of (13), when the fluctuation potential is evaluated around the bounce, is instead given by

$$
\begin{aligned}
\psi_{l s}= & A_{l s} \frac{\rho^{l} R_{s}^{4}}{\left(R_{s}^{2}-\rho^{2}\right)^{2}}\left(1-2\left(\frac{l-1}{l+2}\right) \frac{\rho^{2}}{R_{s}^{2}}+\frac{l(l-1)}{(l+2)(l+3)} \frac{\rho^{4}}{R_{s}^{4}}\right) \\
& +B_{l s} \frac{R_{s}^{l+4}}{\left(R_{s}^{2}-\rho^{2}\right)^{2}} \frac{R_{s}^{l+2}}{\rho^{l+2}} \\
& \times\left(1-2\left(\frac{l+3}{l}\right) \frac{\rho^{2}}{R_{s}^{2}}+\frac{(l+2)(l+3)}{l(l-1)} \frac{\rho^{4}}{R_{s}^{4}}\right) .
\end{aligned}
$$

where $s=1,2$ denotes the two segments of the quarticquartic potential.

On the first segment with $s=1$, regularity of $\psi_{l s}$ at $\rho=0$ requires $B_{l 1}=0$, and we choose the normalization $A_{l 1}=1$, such that we normalize to the $\mathrm{FV}$ at $\rho=0$. This part reduces to the unstable single potential of the SM [30,31], where we can easily read off the ratio $\mathcal{R}_{l}(\infty)=\lim _{\rho \rightarrow \infty} \psi_{l 1} / \rho^{l}$ from the only term remaining in (15) at high $\rho$

$$
\lambda \varphi^{4}: \mathcal{R}_{l}(\infty)=\frac{\psi_{l 1}(\infty)}{\psi_{l}^{\mathrm{FV}}(\infty)}=\frac{l(l-1)}{(l+2)(l+3)} .
$$

On general grounds [80], we expect the $l=0$ mode to be negative, corresponding to the expanding bubble. On the other hand, the four $l=1$ eigenvalues should vanish because of the translational invariance of the center of the bubble (or the bounce solution, which depends only on $\rho$ ). The $\mathcal{R}_{l}(\infty)$ in (16) indeed contains a zero mode at $l=1$, but also has an additional zero at $l=0$, due to the classical scale invariance [30,31].

Let us move on to the second segment and glue the two solutions. The fluctuation potential contains a Dirac delta, therefore the derivative of $\psi_{l}$ changes discontinuously. ${ }^{2}$ The appropriate boundary conditions to join $\psi_{l 1, l 2}$ at $\rho=R_{T}$ are given by

$\psi_{l 1}=\psi_{l 2}, \quad \dot{\psi}_{l 1}=\dot{\psi}_{l 2}+\mu_{V} \psi_{l 1}, \quad \mu_{V}=\frac{\lambda_{1} v_{1}^{3}+\lambda_{2} v_{2}^{3}}{\dot{\bar{\varphi}}\left(R_{T}\right)}$.

These fix the remaining parameters $A_{2 l}, B_{2 l}$ that ultimately determine the behavior of $\mathcal{R}_{l}$ as $\rho \rightarrow \infty$. We arrived to our main result for the fluctuation determinant

$$
\begin{aligned}
\mathcal{R}_{l}(\infty) & =A_{l 2} \frac{l(l-1)}{(l+2)(l+3)} \\
& =\frac{(l-1)\left(l^{3}+c_{2} l^{2}+c_{1} l+c_{0}\right)}{(l+1)(l+2)^{2}(l+3)},
\end{aligned}
$$

with the three coefficients $c_{i}$ that depend only on dimensionless ratios $x$ and $y$ :

$$
\begin{aligned}
& c_{0}=\frac{12(1+x)^{2} x^{4} y\left(1+x^{3} y\right)^{2}}{\left(x^{4} y-1\right)^{3}}, \\
& c_{1}=\frac{2 x\left(1+(1+2 x) x^{2} y\right)\left(2+3 x+(3+4 x) x^{3} y\right)}{\left(x^{4} y-1\right)^{2}}, \\
& c_{2}=\frac{1+4 x+(4+7 x) x^{3} y}{x^{4} y-1} .
\end{aligned}
$$

All $c_{i}$ are real and positive because $x^{4} y>1$, which follows from the construction of the potential. Similarly to the radii $R_{1,2, T}$, the $c_{i}$ diverge in the TW limit.

The zero eigenvalue of the scale invariant single quartic in (16) at $l=0$ is now gone, it got absorbed by the $A_{l 2} \propto l$ in (18). This happens because the quartic-quartic contains mass scales $v_{1,2}$ that break scale invariance, thereby the $l=0$ mode in (18) becomes negative

$$
\mathcal{R}_{0}(\infty)=-\frac{c_{0}}{12}<0,
$$

as required from the instability of the bounce solution.

It follows from (18) that $\mathcal{R}_{l}(\infty) \stackrel{l \gg 1}{\longrightarrow} 1$ and the sum over $l$ in (14) diverges quadratically in the UV-after all, we are

\footnotetext{
${ }^{2}$ Integrating (13) around $R_{T}$, we have $\int_{R_{T}-\epsilon}^{R_{T}+\epsilon} \mathrm{d} \rho \mathcal{O}_{l} \psi_{l}=$ $0 \stackrel{\epsilon \rightarrow 0}{\longrightarrow} \dot{\psi}_{l}\left(R_{T}+\epsilon\right)-\dot{\psi}_{l}\left(R_{T}-\epsilon\right)=-\left(\lambda_{1} v_{1}^{3}+\lambda_{2} v_{2}^{3}\right) \int_{R_{T}-\epsilon}^{R_{T}+\epsilon} \mathrm{d} \rho \delta(\bar{\varphi}(\rho)) \psi_{l}$.
} 
computing a one loop quantity with a tree level counterterm. In Sec. V B we will regularize the sum by subtracting the terms divergent in $l$ and calculate the finite part. Before that, let us deal with the removal of the translational zero eigenvalues of the $l=1$ modes.

\section{B. Removing the zero modes}

As discussed in Sec. II, the prefactor is proportional to the reduced determinant, where the four translational zero eigenvalues are subtracted. The reduced contribution from the $l=1$ modes is defined as

$$
\mathcal{O}_{l} \psi_{l}=\gamma_{n} \psi_{l} \Rightarrow \mathcal{R}_{1}^{\prime}(\infty)=\frac{\prod_{n=2}^{\infty} \gamma_{n}}{\prod_{n=1}^{\infty} \gamma_{n}^{\mathrm{FV}}}
$$

Omitting the zero modes is a straightforward procedure when $\gamma_{n}$ are known for the principal quantum numbers $n$. However, with the Gel'fand-Yaglom approach, the eigenvalues are regrouped in terms of orbital $l$ modes. Thus the zero from translations has to be removed carefully because it multiplies all the other eigenvalues with $l=1$. This can be done perturbatively $[31,75,76,95]$ by off-setting the fluctuation potential with a small dimensionful parameter $\mu_{\varepsilon}^{2}$ and finding the corresponding eigenfunctions of

$$
\left(\mathcal{O}_{1}+\mu_{\varepsilon}^{2}\right) \psi_{1}^{\varepsilon}=0 .
$$

Instead of approaching zero, the ratio of determinants is then given by

$\mathcal{R}_{1}^{\varepsilon}(\infty)=\frac{\psi_{1}^{\varepsilon}(\infty)}{\psi_{1}^{\mathrm{FV}}(\infty)} \simeq \frac{\left(\mu_{\varepsilon}^{2}+\gamma_{1}\right) \prod_{n=2}^{\infty} \gamma_{n}}{\prod_{n=1}^{\infty} \gamma_{n}^{\mathrm{FV}}}=\mu_{\varepsilon}^{2} \mathcal{R}_{1}^{\prime}(\infty)$,

because the $\mu_{\varepsilon}^{2}$ shift does not affect $\gamma_{n>1}$ and $\gamma_{n}^{\mathrm{FV}}$. In other words, we need to compute

$$
\mathcal{R}_{1}^{\prime}(\infty)=\lim _{\mu_{\varepsilon}^{2} \rightarrow 0} \frac{1}{\mu_{\varepsilon}^{2}} \mathcal{R}_{1}^{\varepsilon}(\infty)
$$

The eigenfunctions $\psi_{1}^{\varepsilon}$ are infinitesimally close to $\psi_{1}$ and we can perform a perturbative expansion $\psi_{1}^{\varepsilon} \simeq \psi_{1}+\mu_{\varepsilon}^{2} \delta \psi_{1}$, which enters in (24), such that

$$
\left(\mathcal{O}_{1}+\mu_{\varepsilon}^{2}\right)\left(\psi_{1}+\mu_{\varepsilon}^{2} \delta \psi_{1}\right) \simeq \mathcal{O}_{1} \psi_{1}+\mu_{\varepsilon}^{2}\left(\psi_{1}+\mathcal{O}_{1} \delta \psi_{1}\right)=0 .
$$

The general solution $\psi_{l s}$ in (15) is singular for $l=1$, so we rederive it

$$
\begin{aligned}
\psi_{1 s}= & \frac{R_{s}^{4} \rho}{\left(R_{s}^{2}-\rho^{2}\right)^{2}}\left(A_{1 s}+B_{1 s}\left(\frac{\rho^{4}}{R_{s}^{4}}-8 \frac{\rho^{2}}{R_{s}^{2}}\right.\right. \\
& \left.\left.+24 \log \rho+8 \frac{R_{s}^{2}}{\rho^{2}}-\frac{R_{s}^{4}}{\rho^{4}}\right)\right) .
\end{aligned}
$$

On the first segment with $s=1$, the $\psi_{11}$ needs to be regular at $\rho=0$ and normalized to the $\mathrm{FV}$, therefore $A_{11}=1$ and $B_{11}=0$. Matching to the second segment at $R_{T}$ gives $A_{12}=x^{6} y^{2}$ and $B_{12}=0$. The value at infinity is then given by $\psi_{12}(\infty) \propto B_{12}=0$, as it should be since we are looking at the zero eigenvalue and $\mathcal{R}_{1}(\infty) \propto \psi_{12}(\infty)=0$.

Now that we have the $l=1$ fluctuation, let us move on to perturbations $\delta \psi_{1 s}$, given by the nonhomogeneous equation $\mathcal{O} \delta \psi_{1}=-\psi_{1}$ that comes from (27) and get

$$
\begin{aligned}
\delta \psi_{1 s}= & \frac{3 R_{s}^{6} \rho}{4\left(R_{s}^{2}-\rho^{2}\right)^{2}}\left(\delta A_{1 s}+\frac{\delta B_{1 s}}{18}\left(\frac{\rho^{4}}{R_{s}^{4}}-8 \frac{\rho^{2}}{R_{s}^{2}}\right.\right. \\
& \left.+24 \log \rho+8 \frac{R_{s}^{2}}{\rho^{2}}-\frac{R_{s}^{4}}{\rho^{4}}\right) \\
& \left.-\frac{A_{1 s}}{18}\left(6 \frac{\rho^{2}}{R_{s}^{2}}-18-24 \log \rho-\frac{R_{s}^{2}}{\rho^{2}}+\frac{R_{s}^{4}}{\rho^{4}}\right)\right) .
\end{aligned}
$$

The boundary conditions $\delta \psi_{11}(0)=\dot{\delta \psi_{11}}(0)=0$ fix $\delta A_{11}=$ $\delta B_{11}=-1$ on the first segment ${ }^{3}$ and the same matching conditions required for $\psi_{l}$ in (17), apply to $\delta \psi_{l}$. These determine the remaining $\delta A_{12}$ and $\delta B_{12}=3 \lambda /\left(8 \pi^{2}\right) \mathcal{S}_{0} x^{6} y^{2}$.

In fact, it is $\delta B_{12}$ that gives the reduced determinant after plugging the expansion in (26)

$$
\begin{aligned}
\mathcal{R}_{1}^{\prime}(\infty) & =\left.\lim _{\mu_{\varepsilon}^{2} \rightarrow 0} \frac{1}{\mu_{\varepsilon}^{2}} \frac{\psi_{1}+\mu_{\varepsilon}^{2} \delta \psi_{1}}{\psi_{\mathrm{FV} 1}}\right|_{\rho=\infty}=\left.\frac{\delta \psi_{1}}{\psi_{\mathrm{FV} 1}}\right|_{\rho=\infty}=\frac{R_{2}^{2}}{24} \delta B_{12} \\
& =\frac{R_{2}^{2}}{24}\left(\frac{3 \lambda}{8 \pi^{2}}\right) \mathcal{S}_{0} x^{6} y^{2}
\end{aligned}
$$

where we used the fact that $\psi_{1}(\infty)=0$ and $R_{2}$ was calculated in (8). Note that the $\mathcal{R}_{1}^{\prime}$ is proportional to $\mathcal{S}_{0}$, which cancels with the prefactor in (2). The reduced determinant has the correct dimension of mass ${ }^{-2}$, set by the dimensional $R_{2}$, which in turn is proportional to $1 / v$, the energy scale of the model. The dimensionless $\delta B_{12}$ serves as the numerical pre-factor that diverges in the TW limit and gives an additional suppression to the rate. With the $l=1$ zero removed, we can proceed to the finite part.

\section{Finite sum}

With $\mathcal{R}_{l}$ in (14) at hand, the finite part can be computed in some generality. Let us consider a generic form of $\mathcal{R}_{l}$, given by a ratio of polynomials of order $n$

$$
\mathcal{R}_{l}(\infty)=\prod_{i=1}^{n} \frac{l+1-a_{i}}{l+1-b_{i}}
$$

\footnotetext{
${ }^{3}$ The single quartic case $\lim _{\rho \rightarrow \infty} \delta \psi_{11} / \rho=-R^{2} / 24$ becomes consistent with the SM [30,31] after flipping the sign of $V^{\prime \prime}$, because we assumed $\lambda>0$.
} 
which covers the two cases in (16) and (18). The number of roots and poles must be the same, a consequence of the normalization to the FV in (14). To get the finite part of (14), we first find the asymptotic behavior of $\mathcal{R}_{l}$ by expanding the $\log$ of the determinant for large $l$. The degeneracy factor goes as $l^{2}$, therefore the $\ln \mathcal{R}_{l}$ has to be expanded up to $1 / l^{3}$ to account for the quadratic, linear and log divergencies.

It turns out that the asymptotics of the zeta function, used for renormalization, will be given in powers of $\nu=l+1$, therefore it is convenient to define $\mathcal{R}_{l}^{a}$ by expanding (14) in $1 / \nu$ up to $\mathcal{O}\left(\nu^{-3}\right)$. This is subtracted from (14) and we get

$$
\Sigma_{f}=\sum_{\nu=1}^{\infty} \nu^{2}\left(\ln \mathcal{R}_{l}(\infty)-\ln \mathcal{R}_{l}^{a}(\infty)\right)
$$

which is convergent and can be computed ${ }^{4}$ in a closed form

$$
\begin{aligned}
\Sigma_{f}= & \sum_{i=1}^{n}\left(\frac{a_{i}^{3}}{3} \gamma_{E}-\frac{a_{i}}{12}\left(1+3 a_{i}-6 a_{i}^{2}\right)-\zeta_{R}^{\prime}\left(-2,3-a_{i}\right)-2 a_{i} \zeta_{R}^{\prime}\left(-1,3-a_{i}\right)-a_{i}^{2} \zeta_{R}^{\prime}\left(0,3-a_{i}\right)-(a \rightarrow b)\right) \\
& +\ln \mathcal{R}_{0}(\infty)+4 \ln \mathcal{R}_{1}^{\prime}(\infty) .
\end{aligned}
$$

Here, $\zeta_{R}^{\prime}(s, a)$ is the derivative over $s$ of the generalized Riemann zeta function and $\gamma_{E}$ is the Euler's constant. The three roots $a_{i}$ of the polynomial in (18) are

$$
a_{i}=1-\left(c_{2}+\chi_{i}\left(c_{2}^{2}-3 c_{1}\right) / \theta+\chi_{i}^{*} \theta\right) / 3,
$$

with $\theta^{3}=9 / 2\left(c_{1} c_{2}-3 c_{0}-2 / 9 c_{2}^{3}+\sqrt{\left(27 c_{0}^{2}+4 c_{1}^{3}-18 c_{0} c_{1} c_{2}-c_{1}^{2} c_{2}^{2}+4 c_{0} c_{2}^{3}\right) / 3}\right)$ and $\chi=\{-1,(1 \pm i \sqrt{3}) / 2\}$, while $c_{i}$ are given in (19)-(21). This completes the finite part of the decay rate. Next, we are going to recover the asymptotic terms $\mathcal{R}_{l}^{a}$ that were subtracted in (32) using the zeta function regularization.

\section{ZETA FUNCTION REGULARIZATION}

The decay rate in (2) is a physical quantity that depends on the parameters of the potential $V(\varphi)$ in $S[\varphi]$. These need to be renormalized to make connections between measurements, such as decay rates and scattering cross sections, observed at the minimum of the potential. Most commonly, the renormalization is done perturbatively via Feynman diagrams and dimensional regularization. It introduces an arbitrary renormalization scale $\mu$ to keep the mass dimensions for any $D$ and ascribes $1 /(4-D)$ poles to divergent parts of the momentum integrals. Within a chosen subtraction scheme, such as $\overline{\mathrm{MS}}$, on-shell or other, the renormalized parameters (or counterterms) will remove infinities in physical quantities.

The above holds for the FV decay rate in (2) as well [86]. One can compute the UV part of the determinant with Feynman diagrams $[31,64,65,75,76,96]$ for scalars, fermions and gauge bosons. The counterterms used for other processes, will also make the effective action and therefore the rate, finite. For the effective action, which describes the UV part of the FV decay rate, to be

\footnotetext{
${ }^{4}$ Technically, we do the sum over $\mathcal{R}_{l}(\infty)$ from $\nu=3$ up to a large finite regulator to skip the $l=0,1$ modes, which are then added by hand. The sum over $\mathcal{R}_{l}^{a}(\infty)$ starts from $\nu=1$ as in the renormalization procedure. After the summation, the regulator disappears and can be taken to infinity.
}

consistent with the finite sum over the eigenvalues, the asymptotic parts are computed by expanding in terms of $V^{\prime \prime}(\rho)$ insertions. In the SM this is equivalent to insertions of the quartic and gauge couplings, which defines the power counting.

Alternatively, the UV part of the determinant can be defined by the zeta function [97,98], see [99] for a review. ${ }^{5}$ The zeta function formalism was applied to FV decay in the early works [71] and more recently in [84]. We will review its introduction via the contour integral [100,101,103-105] in the following section. Similarly to dimensional regularization, the renormalization scale is introduced for dimensional reasons to define the zeta function for any value of its argument. As with Feynman diagrams, the UV part is computed perturbatively in powers of $V^{\prime \prime}$. However, contrary to the diagrammatic approach, the UV part of zeta is an expansion in powers of $l$ and therefore serves as a convenient UV regulator. Finally, the renormalization is performed by an analytic continuation of the zeta function and follows from the analyticity of the Riemann zeta function. We will see that the final result for the single quartic rate via Feynman diagrams agrees with the zeta function approach.

\footnotetext{
${ }^{5}$ For a pedagogical introduction with examples regarding the use of spectral functions/functional determinants in physical settings, see [33,100-102].
} 


\section{A. Zeta function via contour integral}

Let us begin by redefining the sum over the eigenvalues of $\mathcal{O}$ in terms of the zeta function

$$
\begin{aligned}
\ln \operatorname{det} \mathcal{O} & =\sum_{\mathbf{n}} \ln \gamma_{\mathbf{n}}=-\left.\frac{\mathrm{d}}{\mathrm{d} s} \sum_{\mathbf{n}}\left(\frac{\mu^{2}}{\gamma_{\mathbf{n}}}\right)^{s}\right|_{s=0} \\
& =-\left.\frac{\mathrm{d}}{\mathrm{d} s}\left(\mu^{2 s} \zeta_{\mathcal{O}}(s)\right)\right|_{s=0},
\end{aligned}
$$

where $\mathbf{n}$ stands for all the quantum numbers and $\mu$ is the renormalization scale, which keeps the sum over eigenvalues dimensionless for all values of $s$. As found in [84], it corresponds to the same scale arising from dimensional regularization in the $\overline{\mathrm{MS}}$ scheme [64]. The zeta function associated to the ratio of determinants is given by the difference

$\zeta=\mu^{2 s}\left(\zeta_{\mathcal{O}}-\zeta_{\mathcal{O}_{\mathrm{FV}}}\right) \quad$ and $\quad \ln \left(\frac{\operatorname{det} \mathcal{O}}{\operatorname{det} \mathcal{O}_{\mathrm{FV}}}\right)=-\left.\frac{\mathrm{d}}{\mathrm{d} s} \zeta(s)\right|_{s=0}$.

The sum over eigenvalues in (35) converges if $\operatorname{Re}(s)>$ $D / 2$ [106]. However, to analytically continue $\zeta$ to the region of interest $s=0$, we have to regularize the integral.

To obtain the analytical structure of $\zeta$ in the range $\operatorname{Re}(s) \leq 2$, we rewrite the sum in (35) as a contour integral. For this purpose, let us consider $\mathcal{O} \psi(\rho, \gamma)=\gamma \psi(\rho, \gamma)$, where $\gamma$ is a continuous complex parameter. The $\psi(\gamma)$ is a generalization of $\psi_{\mathbf{n}}$ in the sense that when the boundary conditions in (11) are imposed, $\gamma$ becomes quantized and $\psi_{\mathbf{n}}$ is recovered with $\gamma \rightarrow \gamma_{\mathbf{n}}$. Now the zeta function is defined as a contour integral

$$
\zeta_{\mathcal{O}}=\sum_{\mathbf{n}} \frac{1}{\gamma_{\mathbf{n}}^{s}}=\frac{1}{2 \pi i} \oint \frac{\mathrm{d} \gamma}{\gamma^{s}} \frac{\mathrm{d}}{\mathrm{d} \gamma} \ln \psi(\infty, \gamma)
$$

The sum over eigenvalues $\gamma_{\mathbf{n}}^{-s}$ is recovered because the simple poles are set by $\mathrm{d} \ln \psi / \mathrm{d} \gamma=\psi^{\prime} / \psi$ and the boundary condition $\psi(\infty, \gamma) \stackrel{\gamma \rightarrow \gamma_{\mathrm{n}}}{\longrightarrow} 0$. Thus, by the residue theorem, the integral in (37) sums up all the eigenvalues, as long as the integration contour runs counterclockwise and encloses the entire real axis, as shown by the solid red line in Fig. 1.

As explained above, we have to deform the contour from the positive real axis, which encloses all the eigenvalues, to the negative one. For this purpose, we split the contour in two paths, parametrized in the complex plane by $\exp ( \pm i \theta) \gamma$. As shown in Fig. 1, we start with a path that runs along the positive real axis,

$$
\begin{aligned}
\zeta_{\mathcal{O}}= & \frac{1}{2 \pi i}\left(\int_{0}^{\infty} \mathrm{d} \gamma \frac{e^{i \theta s}}{\gamma^{s}} \frac{\mathrm{d}}{\mathrm{d} \gamma} \ln \psi\left(\infty, e^{-i \theta} \gamma\right)\right. \\
& \left.+\int_{\infty}^{0} \mathrm{~d} \gamma \frac{e^{-i \theta s}}{\gamma^{s}} \frac{\mathrm{d}}{\mathrm{d} \gamma} \ln \psi\left(\infty, e^{i \theta} \gamma\right)\right)
\end{aligned}
$$

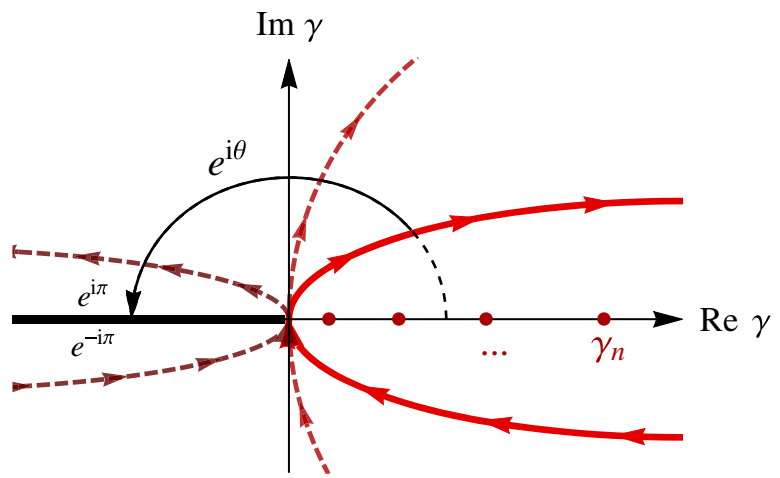

FIG. 1. Deformation of the integration contour in (37) from the positive real axis to the negative one. The red dots represent the location of the poles, such that $\psi(\infty, \gamma)=0$.

and deform it to the negative real axis by taking the limit $\theta \rightarrow \pi$. We get

$$
\zeta_{\mathcal{O}}=\frac{\sin \pi s}{\pi} \int_{0}^{\infty} \frac{\mathrm{d} \gamma}{\gamma^{s}} \frac{\mathrm{d}}{\mathrm{d} \gamma} \ln \psi(\infty,-\gamma),
$$

where we assume that $\psi$ is continuous around the negative real axis, such that $\psi\left(\rho, e^{ \pm i \pi} \gamma\right)=\psi(\rho,-\gamma)$. Finally, since we are considering a hyperspherically symmetric potential, we can separate the variables

$$
\mathcal{O}_{l} \psi_{l}(\rho, \gamma)=\gamma \psi_{l}(\rho, \gamma)
$$

and take into account the degeneracy of the orbital modes. Using (36) and (39), the zeta function for the ratio of determinants is

$$
\zeta=\frac{\sin \pi s}{\pi} \mu^{2 s} \sum_{\nu} \nu^{2} \int_{0}^{\infty} \frac{\mathrm{d} \gamma}{\gamma^{s}} \frac{\mathrm{d}}{\mathrm{d} \gamma} \ln \left(\frac{\psi_{l}(\infty,-\gamma)}{\psi_{l}^{\mathrm{FV}}(\infty,-\gamma)}\right) .
$$

Alas, a closed form solution of $\psi_{l}(\rho, \gamma)$ cannot be obtained in general, even for the single quartic potential. Since we are only interested in the asymptotic behavior $\psi_{l}(\infty, \gamma)$ near the $\mathrm{FV}$, it is enough to consider the expansion around the FV, where the solution of (40) is

$$
\psi_{l}^{\mathrm{FV}}(\rho,-\gamma)=I_{\nu}(\sqrt{\gamma} \rho) / \rho
$$

The $K_{\nu}$ part is discarded due to regularity at $\rho=0$ and the normalization factor is chosen to be one. When $\rho \rightarrow \infty$, the fluctuation potential approaches the $\mathrm{FV}^{6}$ and we can set up an approximate solution

$$
\psi_{l}(\rho,-\gamma) \simeq f_{l}(\gamma) \psi_{l}^{\mathrm{FV}}(\rho,-\gamma),
$$

\footnotetext{
${ }^{6}$ In general, one should subtract $V_{\mathrm{FV}}^{\prime \prime}$ from $V^{\prime \prime}$ and shift the eigenvalues $\gamma \rightarrow \gamma-V_{\mathrm{FV}}^{\prime \prime}$ in (39) and (40), modifying the lower limit of integration. For quartic potentials $V_{\mathrm{FV}}^{\prime \prime}=0$.
} 
where $f_{l}(\gamma)$ is a constant to be determined in the section below. The $K_{\nu}$ term was neglected, because it vanishes in the asymptotic limit. With this ansatz, (41) becomes

$$
\zeta=\frac{\sin \pi s}{\pi} \mu^{2 s} \sum_{\nu} \nu^{2} \int_{0}^{\infty} \frac{\mathrm{d} \gamma}{\gamma^{s}} \frac{\mathrm{d}}{\mathrm{d} \gamma} \ln f_{l}(\gamma),
$$

which is valid for $\operatorname{Re}(s)>2$. In order to make it well defined around $s=0$, we have to find the asymptotic form of $f_{l}$ and renormalize it.

\section{B. Renormalization of the functional determinant}

To perform the analytical continuation of $\zeta$ to $s=0$, we define its asymptotic limit by expanding (44) in the large $l$ limit

$$
\zeta_{a}=\frac{\sin \pi s}{\pi} \mu^{2 s} \sum_{\nu} \nu^{2} \int_{0}^{\infty} \frac{\mathrm{d} \gamma}{\gamma^{s}} \frac{\mathrm{d}}{\mathrm{d} \gamma} \ln f_{l}^{a}(\gamma),
$$

and compute $f_{l}^{a}$ perturbatively by expanding around the $\mathrm{FV}$. Once we have $\zeta_{a}$, we subtract it from $\zeta$, which removes the leading $l$ divergence and produces the finite result

$$
\zeta_{f}=\zeta-\zeta_{a}
$$

similarly to what was done for the finite sum in Sec. IV C. Finally, the divergent terms in $\zeta_{a}$ will be renormalized using the analytic properties of the Riemann zeta function.

\section{Asymptotic expansion of the zeta}

As discussed above, we would like to compute (45) by considering a double expansion. First, $\rho \rightarrow \infty$ in (41), which allows us to construct an implicit iterative solution around the FV for a fixed angular mode $l$. Then the high- $l$ expansion can be performed and we end up with a closed form expression for $\zeta_{a}$.

False vacuum expansion. When approaching the FV, (40) can be solved by starting from $\psi_{l}^{\mathrm{FV}}(\rho,-\gamma)$, given by (42), and writing down the general ansatz

$\psi_{l}(\rho,-\gamma)=\psi_{l}^{\mathrm{FV}}(\rho,-\gamma)+\int_{0}^{\rho} \mathrm{d} \rho_{1} G\left(\rho, \rho_{1}\right) V^{\prime \prime}\left(\rho_{1}\right) \psi_{l}\left(\rho_{1},-\gamma\right)$,

$G\left(\rho, \rho_{1}\right)=\frac{\rho_{1}^{2}}{\rho}\left(I_{\nu}(\sqrt{\gamma} \rho) K_{\nu}\left(\sqrt{\gamma} \rho_{1}\right)-I_{\nu}\left(\sqrt{\gamma} \rho_{1}\right) K_{\nu}(\sqrt{\gamma} \rho)\right)$,

where $G$ is the Green function associated with $\mathcal{O}_{l}$. This is a self-referential integral equation, which can be solved iteratively by starting in the FV and expanding in powers of $V^{\prime \prime}$. The iteration stops when the zeta function becomes well defined in the asymptotic UV limit and describes all the high $l$ modes.

Actually, we already know from the normalization in (14), and the discussion regarding the finite sum in Sec. IV C, that the asymptotic terms need to go up to $\nu^{-3}$. In the doubly asymptotic limit when $\rho, \nu \rightarrow \infty$, the Green function is proportional to $1 / \nu$, which follows from the properties of Bessel functions in the Appendix A. Thus, each insertion of $V^{\prime \prime}$ in (47) comes with a factor of $1 / \nu$ and it is enough to expand the zeta up to $\mathcal{O}\left(V^{\prime \prime 3}\right)$. Using (43) and (47), we have

$$
\begin{aligned}
\frac{\psi_{l}(\infty,-\gamma)}{\psi_{l}^{\mathrm{FV}}(\infty,-\gamma)} & =f_{l}(\gamma) \\
& =1+\int_{0}^{\infty} \mathrm{d} \rho \rho^{2} K_{\nu}(\sqrt{\gamma} \rho) V^{\prime \prime}(\rho) \psi_{l}(\rho,-\gamma) \\
& =1+f_{l}^{(1)}+f_{l}^{(2)}+f_{l}^{(3)}+\mathcal{O}\left(V^{\prime \prime 4}\right)
\end{aligned}
$$

while expanding the log to the same order gives

$$
\begin{aligned}
\ln f_{l}(\gamma) \simeq & f_{l}^{(1)}-\frac{1}{2}\left(f_{l}^{(1) 2}-2 f_{l}^{(2)}\right) \\
& +\frac{1}{3}\left(f_{l}^{(1) 3}-3 f_{l}^{(1)} f_{l}^{(2)}+3 f_{l}^{(3)}\right) .
\end{aligned}
$$

The integrals $f_{l}^{(n)}$ are obtained by iterating (47)

$$
\begin{aligned}
f_{l}^{(1)}= & \int_{0}^{\infty} \mathrm{d} \rho_{1} \rho_{1} V^{\prime \prime}\left(\rho_{1}\right) K_{\nu}\left(\sqrt{\gamma} \rho_{1}\right) I_{\nu}\left(\sqrt{\gamma} \rho_{1}\right) \\
f_{l}^{(2)}= & \int_{0}^{\infty} \mathrm{d} \rho_{1} \rho_{1}^{2} V^{\prime \prime}\left(\rho_{1}\right) K_{\nu}\left(\sqrt{\gamma} \rho_{1}\right) \\
& \times \int_{0}^{\rho_{1}} \mathrm{~d} \rho_{1} G_{12} V^{\prime \prime}\left(\rho_{2}\right) \frac{I_{\nu}\left(\sqrt{\gamma} \rho_{2}\right)}{\rho_{2}} \\
f_{l}^{(3)}= & \int_{0}^{\infty} \mathrm{d} \rho_{1} \rho_{1}^{2} V^{\prime \prime}\left(\rho_{1}\right) K_{\nu}\left(\sqrt{\gamma} \rho_{1}\right) \int_{0}^{\rho_{1}} \mathrm{~d} \rho_{1} G_{12} V^{\prime \prime}\left(\rho_{2}\right) \\
& \times \int_{0}^{\rho_{2}} \mathrm{~d} \rho_{2} G_{23} V^{\prime \prime}\left(\rho_{3}\right) \frac{I_{\nu}\left(\sqrt{\gamma} \rho_{3}\right)}{\rho_{3}},
\end{aligned}
$$

where $G_{i j}=G\left(\rho_{i}, \rho_{j}\right)$. This concludes the FV expansion in $V^{\prime \prime}$ and we can focus on isolating the high- $l$ behavior.

High-l expansion. We would like to expand $f_{l}^{(i)}$ for high $l$ up to $\mathcal{O}\left(\nu^{-3}\right)$, while keeping $\rho \rightarrow \infty$. To this end, we evaluate the Bessel functions in (52)-(54) in the limit when $\nu, \rho \rightarrow \infty$ with $\sqrt{\gamma} \rho / \nu$ fixed, and use the saddle point approximation, see (A1)-(A3) in the Appendix A for technical details.

For continuous $V^{\prime \prime}$, the integrals in (52)-(53) were calculated by $[65,84]$ and (54) was not needed. Here, we extend the analysis to take into account the delta function 
$V^{\prime \prime}(\rho)=\sum_{s} V_{s}^{\prime \prime}(\rho) H\left((-1)^{s}\left(\rho-R_{T}\right)\right)-\mu_{V} \delta\left(\rho-R_{T}\right)$.

Performing the integrals (52)-(54) requires some effort and we leave the details to the Appendix B. The final result up to $\mathcal{O}(\sqrt{\gamma} / \nu)^{4}$ is fairly compact

$$
\begin{aligned}
\ln f_{l}^{a}= & \sum_{s} \int_{0}^{\infty} \mathrm{d} \rho \rho V_{s}^{\prime \prime}\left(\frac{t}{2 \nu}+\frac{t^{3}}{16 \nu^{3}}\left(1-6 t^{2}+5 t^{4}-2 \rho^{2} V_{s}^{\prime \prime}\right)\right) \\
& \times H\left((-1)^{s}\left(\rho-R_{T}\right)\right) \\
& -\mu_{V} R_{T}\left(\frac{t}{2 \nu}+\frac{t^{3}}{16 \nu^{3}}\left(1-6 t^{2}+5 t^{4}\right)+\mu_{V} R_{T} \frac{t^{2}}{8 \nu^{2}}\right. \\
& \left.+\left(\mu_{V} R_{T}\right)^{2} \frac{t^{3}}{24 \nu^{3}}\left(1-\frac{3}{\mu_{V}^{2}}\left(V_{1}^{\prime \prime}+V_{2}^{\prime \prime}\right)\right)\right)\left.\right|_{\rho=R_{T}}
\end{aligned}
$$

where $t=\left(1+\gamma(\rho / \nu)^{2}\right)^{-1 / 2}$. The first line corresponds to the continuous part of $V^{\prime \prime}$ and reproduces the known results of [84] when $V_{\mathrm{FV}}^{\prime \prime}=0$. The terms proportional to $\mu_{V}$ are new because of the presence of the delta at $R_{T}$. This completes the asymptotic description of zeta and (56) can be used to evaluate the finite sum and carry out the renormalization.

\section{Regularization of the finite zeta}

The asymptotic form of the zeta function allows us to regulate the large $l$ infinities and compute the finite sum, similarly to what we did in §IV C. From (45) and (46), we have

$\zeta_{f}=\frac{\sin \pi s}{\pi} \sum_{\nu} \nu^{2} \mu^{2 s} \int_{0}^{\infty} \frac{\mathrm{d} \gamma}{\gamma^{s}} \frac{\mathrm{d}}{\mathrm{d} \gamma}\left(\ln f_{l}(\gamma)-\ln f_{l}^{a}(\gamma)\right)$,

which is finite and analytic in the neighborhood of $s=0$. This means we can take the derivative with respect to $s$ and evaluate $\zeta_{f}^{\prime}(0)$. In doing that, the terms proportional to $\sin \pi s$ vanish, $\gamma^{-s}$ goes to one and the integral can be computed trivially by evaluating the terms on the boundaries.

On the upper limit $\gamma \rightarrow \infty$ and $V^{\prime \prime}(\rho)$ in (40) vanishes, thus $\psi_{l}(\rho, \gamma)$ goes to the FV solution and $f_{l}(\gamma \rightarrow \infty) \rightarrow 1$ for both log terms in (57), which go to zero. This leaves us with the two terms on the lower boundary, when $\gamma \rightarrow 0$ (and $\rho \rightarrow \infty$, as usual). First, from the definition of $f_{l}(\gamma)$ in (43) and from (40), it becomes clear that we end up with the same equation (13) that defined $\mathcal{R}_{l}(\infty)$. In other words, $f_{l}(0)=\mathcal{R}_{l}(\infty)$. Second, we need to evaluate the asymptotic part $f_{l}^{a}(0)$ by setting $\gamma=0$ in (56) which sets $t=1$ and we can integrate over $\rho$ for a specific fluctuation potential. Now, the finite sum can be performed and we reproduce $\Sigma_{f}$ in (32), such that

$$
-\zeta_{f}^{\prime}(0)=\sum_{\nu} \nu^{2}\left(\ln \mathcal{R}_{l}(\infty)-\ln f_{l}^{a}(0)\right)=\Sigma_{f}
$$

for the single and the quartic-quartic potential. As a very nontrivial cross-check of the asymptotics, we find that $f_{l}^{a}$ computed from (56), which is defined directly in terms of $V^{\prime \prime}$, is precisely equal to the one from $\mathcal{R}_{l}(\infty)$ in (31), i.e., $f_{l}^{a}(0)=\mathcal{R}_{l}^{a}(\infty)$.

The procedure that gave (58) does not always reproduce the finite $\operatorname{sum} \Sigma_{f}$. In particular, when $V_{\mathrm{FV}}^{\prime \prime} \neq 0$, the lower limit of integration over $\gamma$ is shifted from 0 to $\sqrt{V_{\mathrm{FV}}^{\prime \prime}}$, and we have to evaluate $f_{l}^{a}\left(\sqrt{V_{\mathrm{FV}}^{\prime \prime}}\right)$. In this case, additional terms appear in (56) because $t \neq 1$. However, this is an oversubtraction [84] — such terms are suppressed by $1 / \nu^{4}$ or more and get canceled by the renormalized parts below.

\section{Renormalization of the asymptotic zeta}

The asymptotic part of the zeta function can now be renormalized. The integrals in (56) are evaluated using the following identity, valid for $\operatorname{Re}(s)<1$

$$
\frac{\sin \pi s}{\pi} \mu^{2 s} \int_{0}^{\infty} \frac{\mathrm{d} \gamma}{\gamma^{s}} \frac{\mathrm{d}}{\mathrm{d} \gamma} t^{n}=-\frac{\Gamma\left(s+\frac{n}{2}\right)(\mu \rho)^{2 s}}{\Gamma(s) \Gamma\left(\frac{n}{2}\right)} \nu^{-2 s} .
$$

The resulting expressions are plugged into (45) and we perform the sum over $\nu$. Each term that goes as $(t / \nu)^{n}$ gives a Riemann zeta $\zeta_{R}(2 s+n-2)$. The analytic continuation properties of $\zeta_{R}$ are well known and provide a mathematical description of divergencies. Finally, we take the derivative over $s$ and send $s$ to zero, ending up with

$$
\begin{aligned}
\zeta_{a}^{\prime}(0)= & \sum_{s} \frac{1}{8} \int_{0}^{\infty} \mathrm{d} \rho \rho^{3} V_{s}^{\prime \prime 2}\left(\ln \left(\frac{\mu \rho}{2}\right)+\gamma_{E}+1\right) \\
& \times H\left((-1)^{s}\left(\rho-R_{T}\right)\right) \\
& -\frac{\left(\mu_{V} R_{T}\right)^{2}}{16}+\frac{\left(\mu_{V} R_{T}\right)^{3}}{24}\left(1-\left.\frac{3}{\mu_{V}^{2}}\left(V_{1}^{\prime \prime}+V_{2}^{\prime \prime}\right)\right|_{R_{T}}\right) \\
& \times\left(\ln \left(\frac{\mu R_{T}}{2}\right)+\gamma_{E}+1\right)
\end{aligned}
$$

This agrees with [84] for a continuous $V^{\prime \prime}$ with $\mu_{V}=0$ and $V_{\mathrm{FV}}^{\prime \prime}=0$ and also reproduces the SM [31] when applied to the single quartic. This also demonstrates that the Feynman diagrammatic approach coincides with the zeta function formalism. Another nontrivial check regards the cancellation of divergences, i.e., we verify that terms proportional to $\gamma_{E}$ in $\Sigma_{f}$ given by (33) cancel the ones in $\zeta_{a}^{\prime}(0)$ above.

Let us comment on the renormalization scale dependence. The FV decay is a physical process and the rate should not depend on $\mu$. Specifically, the $\mu$ dependence from the prefactor cancels the $\ln \mu$ from running of parameters in the bounce action $\mathcal{S}_{0}$. For the single quartic case this is easy to see. The first segment gives $1 / 2 \zeta_{a}^{\prime}(0) \supset 3 \ln \mu$, while the running of the quartic $\beta_{\lambda}=\mathrm{d} \lambda / \mathrm{d} \ln \mu=9 \lambda^{2} /\left(8 \pi^{2}\right)$ is solved for $\lambda(\mu)$ and plugged into the bounce action $-8 \pi^{2} /(3 \lambda(\mu))$ to cancel the $\mu$ dependence of the prefactor. While running the 
quartic-quartic potential couplings is beyond the scope of this work, we confirm that the leading order running of $\lambda_{1,2}$ with the above beta functions cancels the $\mu$ dependence of the continuous part of (60) in the weakly coupled limit when $x$ and $y$ are small.

\section{SUMMARY OF DECAY RATES}

The final result for the renormalized log of the functional determinant is

$$
\ln \left(\frac{\operatorname{det} \mathcal{O}}{\operatorname{det} \mathcal{O}_{\mathrm{FV}}}\right)=-\zeta^{\prime}(0)=-\zeta_{f}^{\prime}(0)-\zeta_{a}^{\prime}(0)
$$

where $-\zeta_{f}^{\prime}(0)=\Sigma_{f}$ can be found in (33) and $\zeta_{a}^{\prime}(0)$ in (60). Therefore, the total decay rate per $4 \mathrm{D}$ unit volume is

$$
\frac{\Gamma}{\mathcal{V}}=\left(\frac{\mathcal{S}_{0}}{2 \pi}\right)^{2} e^{-\mathcal{S}_{0}+\frac{1}{2} \zeta^{\prime}(0)}=v^{4} e^{-\mathcal{S}_{0}-\mathcal{S}_{1}},
$$

where the $\mathcal{S}_{0}$ comes from (9) and $\zeta^{\prime}(0)$ is the sum of (33) and (60). As we will see, having a closed form result is particularly useful to study the behavior of the rate in the TW limit as well as for the large scale separation $x \gg 1$, corresponding to a rather flat potential.

\section{A. Real quartic}

To complete the calculation for the real scalar part, we evaluate the integral in (60) in a closed form with $R_{1,2, T}$ and $\mu_{V}$ given by (8) and (17), respectively. This is a straightforward calculation, but we omit the entire expression for brevity $^{7}$ and instead show the negative log of the normalized rate $-\ln \Gamma / \mathcal{V} / v^{4}$ in Fig. 2. The total rate is shown by the black solid line on Fig. 2 for a fixed $\lambda_{1}=\lambda_{2}=1$ as $x=v_{1} / v_{2}$ interpolates from the TW $x \sim 1$ to the thick wall and a flat potential when $x \gg 1$. We assume that all the couplings are defined at $v$ and set the renormalization scale to $\mu=v$. In this case, the rate is insensitive to $v$, apart from the overall normalization factor $v^{4}$, which is factorized in the plot.

The contribution from the semiclassical action $\mathcal{S}_{0}$ in the first term of (63), coming from the bounce action is shown in dashed red and tends to dominate for small $x$, as long as $\lambda$ is small. The prefactor correction $\mathcal{S}_{1}$ is plotted in dashed yellow. It is subdominant for small values of $x$ and starts to dominate for $x \sim 4$, the rate drops and then rises logarithmically. Strictly speaking, these two are not separate contributions, i.e., running the parameters in $\mathcal{S}_{0}$ will exactly cancel the $\mu$ dependent part of $\mathcal{S}_{1}$. Of course, once this is done, one has to include all the corrections of the same order, in particular the $\log R$ and finite parts-they all contribute with the same power of the coupling constant.

\footnotetext{
${ }^{7}$ We provide a complete Mathematica notebook with the entire calculation as ancillary material [107].
}

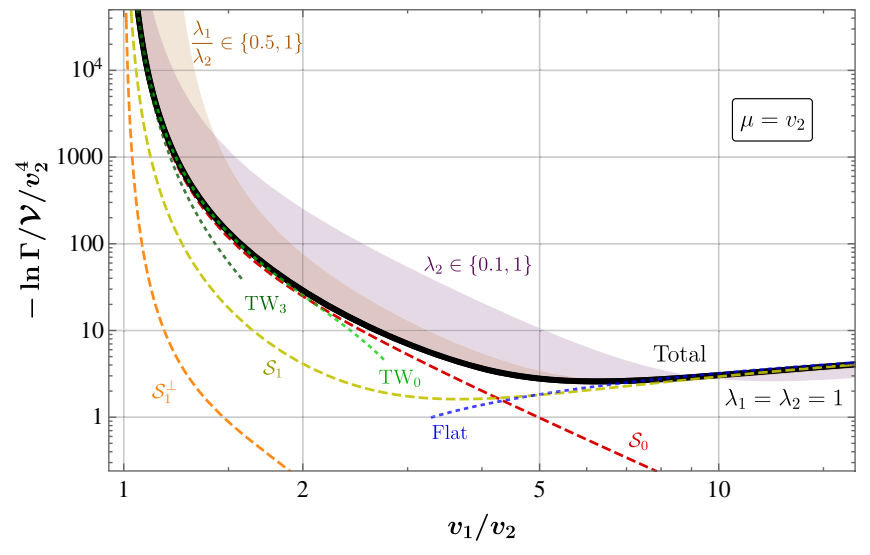

FIG. 2. The FV decay rate for the quartic-quartic potential in (5). The black solid line shows the total rate, while the dashed ones show the semiclassical part $\mathcal{S}_{0}$ in red and the finite renormalized prefactor $\mathcal{S}_{1}$ in dark yellow. The dotted lines correspond to the TW leading expansion, where we set $y=$ $\lambda_{1} / \lambda_{2}=1$ and expand up to $(x-1)^{-3}$ in dark green, additional corrections up to $(x-1)^{0}$ in light green and the flat potential limit $x=v_{1} / v_{2} \gg 1$ in blue. The shaded regions show the variation of $\lambda_{2} \in\{0.1,1\}$ in purple and $\lambda_{1} / \lambda_{2} \in\{0.5,1\}$ in light brown.

The fact that the running of couplings, or alternatively the $\mu$-dependent part of the prefactor, becomes important for large $x$, is not surprising. After all, this is happening in the regime $v_{1}>v_{2}$, where a large separation of scales is present. This is exactly analogous to any quantum field theoretic calculation, where large logs appear when scales are separate and one needs to resum them, even in perturbative theories like quantum electrodynamics.

However, this does not imply a breakdown of the semiclassical approximation - the leading bounce contribution will always dominate for a small enough $\lambda$. In any case, lowering $\lambda_{2}$ results in a higher $\mathcal{S}_{0}$ which dominates the $\mathcal{S}_{1}$ for larger values of $x$, as shown by the purple shaded region. The variation of $y$, on the other hand, results in a shift of the entire curve to larger $x$, as shown by the brown shaded region, because the thin wall pole in the rate happens when $x^{4} y \simeq 1$.

The behavior of the rate simplifies considerably in these two limits. Near the thin wall $x^{4} y \sim 1$ (TW: $x \sim 1+\varepsilon$, $y=1)$, the $a_{i}$ become large and negative, thus the asymptotic expansion of $\zeta^{\prime}\left(s, 3-a_{i}\right)$ in (A4)-(A6) can be used. Conversely, the $a_{i}$ become nearly constant when $x \gg 1$ (flat) and we have

$$
-\ln \frac{\Gamma}{\mathcal{V}} \frac{1}{v^{4}} \simeq \begin{cases}\frac{1}{\varepsilon^{3}}\left(\frac{2 \pi^{2}}{3 \lambda}+\frac{2}{9}+\frac{\pi}{2 \sqrt{3}}-\frac{1}{12} \ln \frac{2 \lambda v^{2}}{\mu^{2}}\right), & \text { TW }, \\ \frac{7}{12}-2 \zeta_{R}^{\prime}(-1)+\frac{1}{3} \ln \frac{y^{2} \lambda^{2} v^{4} x^{6}}{32 \pi^{3} \mu^{4}}, & \text { Flat. }\end{cases}
$$

The leading TW functional dependence goes as $\varepsilon^{-3}$, which is the same as in the TW approximation of the displaced quadratic potential [71], with different numerical 
coefficients and an additional log term. The TW series can easily be extended to arbitrary order in $\varepsilon$; we plot the leading $\varepsilon^{-3}$ and the expansion up to $\varepsilon^{0}$ with the dotted green lines in Fig. 2. These two are fairly good proxies and cover a significant portion of parameter space, as seen from Fig. 2.

\section{B. Complexified quartic}

Let us extend the analysis to the complexified version of the model and examine the effect of transverse fluctuations, coming from the imaginary field component.

As we will see, when restricted to the single quartic, we are dealing with a global $U(1)$ symmetric theory with a massless Goldstone and obtain an additional zero eigenvalue. This situation is somewhat similar to the SM, however one should be careful with the comparison, since the equations of motion and the would-be-Goldstone masses are gauge-dependent $[31,76,108]$. Once we add the second quartic segment, the global $U(1)$ disappears together with the zero eigenvalue.

Consider a complex scalar field $\Phi=\left(\varphi+i \varphi_{\perp}\right) / \sqrt{2}$ and the complexified version of the potential

$$
\begin{aligned}
V(\Phi)= & \left(\lambda_{2} v_{2}^{4}-\lambda_{1} v_{1}^{4}+\lambda_{1}\left|\Phi+v_{1}\right|^{4}\right) H(\tilde{\Phi}-\Phi) \\
& +\lambda_{2}\left|\Phi-v_{2}\right|^{4} H(\Phi-\tilde{\Phi}),
\end{aligned}
$$

where $\tilde{\Phi}(\Phi)$ describes the boundary between the two regions. It is chosen such that $V$ is continuous in the $\varphi-\varphi_{\perp}$ plane and $\tilde{\Phi}$ goes to zero on the $\varphi_{\perp}=0$ axis, reproducing (5). Parameters of the potential are still real and the bounce for the real component $\varphi$ stays the same, as does the determinant.

The perpendicular component $\varphi_{\perp}$ carries no vev, because $v_{1,2} \in \mathbb{R}$, and its bounce is zero. The fluctuations $\psi_{l}^{\perp}$ are nonzero and obey

$$
\begin{aligned}
\mathcal{O}_{\perp} \psi_{l}^{\perp} & =-\ddot{\psi}_{l}^{\perp}-\frac{3}{\rho} \dot{\psi}_{l}^{\perp}+\frac{l(l+2)}{\rho^{2}} \psi_{l}^{\perp}+V_{\perp}^{\prime \prime}(\bar{\varphi}) \psi_{l}^{\perp}=0, \\
V_{\perp}^{\prime \prime} & =\frac{1}{3} V^{\prime \prime} .
\end{aligned}
$$

The FV normalization stays the same $\psi_{l s}^{\mathrm{FV} \perp}=\rho^{l}$, while the transverse fluctuations are simpler than the real scalar ones

$$
\begin{aligned}
\psi_{l s}^{\perp}= & \frac{\rho^{l} R_{s}^{2}}{R_{s}^{2}-\rho^{2}}\left(A_{l s}^{\perp}\left(1-\left(\frac{l}{l+2}\right) \frac{\rho^{2}}{R_{s}^{2}}\right)\right. \\
& \left.+B_{l s}^{\perp} \frac{R_{s}^{2 l+2}}{\rho^{2 l+2}}\left(1-\left(\frac{l+2}{l}\right) \frac{\rho^{2}}{R_{s}^{2}}\right)\right) .
\end{aligned}
$$

The boundary conditions fix $A_{l 1}^{\perp}=1, B_{l 1}^{\perp}=0$, such that dividing by $\rho^{l}$ and taking the limit $\rho \rightarrow \infty$, we recover the single quartic global Goldstone [31]

$$
\lambda|\Phi|^{4}: \mathcal{R}_{l}^{\perp}(\infty)=\frac{l}{l+2},
$$

where the zero eigenvalue at $l=0$ appears due to the $U(1)$ symmetry. Proceeding to the second segment and taking into account the matching conditions, we end up with

$$
\mathcal{R}_{l}^{\perp}(\infty)=A_{l 2}^{\perp} \frac{l}{l+2}=\frac{l^{3}+c_{2}^{\perp} l^{2}+c_{1}^{\perp} l+c_{0}^{\perp}}{(l+1)(l+2)^{2}} .
$$

After adding the second segment, the $U(1)$ symmetry gets broken and the zero eigenvalue in (67) disappears. The coefficients are then given by $c_{0}^{\perp}=c_{0} / 9$ and

$$
\begin{aligned}
c_{1}^{\perp} & =\frac{2 x\left((2 x+1) x^{2} y+1\right)\left((4 x+1) x^{3} y+x-2\right)}{3\left(x^{4} y-1\right)^{2}}, \\
c_{2}^{\perp} & =\frac{(13 x+4) x^{3} y+4 x-5}{3\left(x^{4} y-1\right)} .
\end{aligned}
$$

The $\mathcal{R}_{l}^{\perp}(\infty)$ goes to 1 as $l \gg 1$ and the ratio of determinants diverges. To get the total rate, we proceed as for the real quartic above. The solutions to the cubic polynomial in (68) are given by the same expression in (34) with replacing $c_{i} \rightarrow c_{i}^{\perp}$ and the fluctuation potential $V_{\perp}^{\prime \prime} \rightarrow V^{\prime \prime} / 3$. Again, the asymptotic behavior is simple

$$
\mathcal{S}_{1}^{\perp} \simeq \begin{cases}\frac{1}{972 \varepsilon^{3}}\left(152-8 \ln 27-12 \sqrt{11} \arctan \sqrt{11}-57 \ln \frac{2 \lambda v^{2}}{\mu^{2}}\right), & \mathrm{TW}, \\ \frac{322}{81} \ln 2-\frac{1}{324}-\frac{\zeta_{R}(3)}{8 \pi^{2}}-\frac{5}{2} \ln 3+\frac{2}{81} \ln \frac{\lambda y x^{3} v^{2}}{\mu^{2}}-\frac{\zeta_{R}^{\prime}\left(-2, \frac{7}{3}\right)}{2}-\frac{2 \zeta_{R}^{\prime}\left(-1, \frac{7}{3}\right)}{3}-\frac{2 \zeta_{R}^{\prime}\left(0, \frac{7}{3}\right)}{9}, & \text { Flat },\end{cases}
$$

and the total rate is obtained by adding $\mathcal{S}_{1}^{\perp}$ to $\mathcal{S}_{1}$ in (62). It turns out that the correction from the transverse fluctuations are rather small and subdominant with respect to the real scalar ones, as seen from the orange dashed line on Fig. 2.

\section{CONCLUSIONS AND OUTLOOK}

We presented a closed-form solution for the total decay rate at one loop for a potential with two tree level minima of a quartic-quartic potential. Our approach is based on the Gel'fand-Yaglom theorem that circumvents the need to 
obtain individual eigenvalues of the fluctuation operator. The existing renormalization procedure had to be generalized to include the delta functions in the fluctuation potential. To this end, an appropriate expansion of the fluctuation functions to the maximal $1 / l^{3}$ term had to be performed to extract the UV behavior and regularize the determinant. It might be of interest to reproduce this result with the Feynman diagrammatic approach and also obtain the RGE running of parameters for this particular case.

The final expression for the FV decay rate in (62) consists of the semiclassical action $\mathcal{S}_{0}$ and the finite and renormalized corrections $\mathcal{S}_{1}$. Both are calculated for a complete range of parameters of the potential-thus we get an exact one loop result for the renormalized decay rate. The main result of our work is summarized in Fig. 2, where the behavior of the rate for thin and thick walls becomes apparent, as well as the range of validity of the simple approximations that were derived from the exact result. Perhaps the main take-away message here is that for a large separation of scales, one has to include the running of parameters in $\mathcal{S}_{0}$ and subsequently compute the renormalization scale dependent prefactor, such that the total rate becomes $\mu$-independent. Note that this does not signal the breakdown of perturbativity, which instead is governed by the overall size of the quartic coupling $\lambda$.

We also included the effects of the imaginary component of the complex field, which are found to be subdominant in general. Similarly to the SM, which corresponds to the single quartic, the effect of fermions and gauge bosons could be taken into account. To this end, the known results $[31,75,76]$, for spin $1 / 2$ and 1 fluctuations should be extended to include the second quartic segment while taking into account the presence of Dirac delta, in complete analogy to the imaginary complex scalar.

The present calculation relies on an exact bounce solution that can be found in $D=4$. It may be of interest to extend its validity via dimensional continuation to other dimensions, $D=3$ in particular. This may be possible to do perturbatively, similarly to the bounce [109], near the thin wall, where the $1 / \rho$ term does not play a significant role. Likewise, one may consider other solvable bounces, such as the log potential [42,44], quadratic-quadratic [54], binomial [43] and (extended) polygonal [41]. The latter is particularly interesting because the fluctuation potential is smooth and avoids the delta function. At the same time, it can serve as a universal estimator of the total rate and might be extended to multifields [110], where only recently [70] progress was made.

\section{ACKNOWLEDGMENTS}

We thank Rok Medveš for discussions and work on topics related to this work. The work of V. G. was supported by the Slovenian Research Agency's young researcher program under the Grant No. PR-07582. M. N. was supported by the Slovenian Research Agency under the research core funding
No. P1-0035 and in part by the research grant J1-8137. M. N. acknowledges the support of the COST action CA16201- "Unraveling new physics at the LHC through the precision frontier".

\section{APPENDIX A: BESSEL, SADDLE-POINT AND ZETA FUNCTION APPROXIMATIONS}

\section{Bessel functions}

To perform the high- $l$ expansion in Sec. V B 1, we used the mathematical properties of the Bessel functions that can be found on p. 378 of [111] Eqs. (9.7.7) and (9.7.8). Expanding for large $\nu$ and $\rho$, while keeping $\rho / \nu$ fixed, we have up to $\mathcal{O}\left(\nu^{-4}\right)$

$$
I_{\nu}(\sqrt{\gamma} \rho) K_{\nu}(\sqrt{\gamma} \rho)=\frac{t}{2 \nu}+\frac{t^{3}}{16 \nu^{3}}\left(1-6 t^{2}+5 t^{4}\right),
$$

and up to $\left(1+\mathcal{O}\left(\nu^{-1}\right)\right)$

$$
I_{\nu}^{2}(\sqrt{\gamma} \rho)=\frac{t}{2 \pi \nu} e^{2 \nu \eta}, \quad K_{\nu}^{2}(\sqrt{\gamma} \rho) \sim \frac{\pi t}{2 \nu} e^{-2 \nu \eta},
$$

with $\eta=t^{-1}+\ln \left(\sqrt{\gamma} \rho / \nu /\left(1+t^{-1}\right)\right)$.

Saddle-point approximation can be found on p. 362 of $[100,101]$ Eq. E.14. It can be used to expand the integrals in Sec. V B 1 in powers of $1 / \nu$ when the leading contribution is dominated by the exponential high- $l$ terms from (A2). Expanding up to $\mathcal{O}\left(\nu^{-2}\right)$

$$
\int_{0}^{\rho} \mathrm{d} \rho_{1} f\left(\rho_{1}\right) e^{\nu B\left(\rho_{1}\right)}=e^{\nu B(\rho)} \frac{f(\rho)}{\nu}\left(\frac{\mathrm{d} B(\rho)}{\mathrm{d} \rho}\right)^{-1} .
$$

\section{Generalized Riemann zeta function}

A useful asymptotic expansion of the derivatives of the generalized zeta function, is applicable in the TW limit $a \gg 1$ and can be found in Eqs. (18) and (19) of [112]

$$
\begin{aligned}
\zeta_{R}^{\prime}(0, a)= & \ln \Gamma(a)-\frac{\ln 2 \pi}{2} \sim-a+a \log a-\frac{\log a}{2}, \\
\zeta_{R}^{\prime}(-1, a) \sim & -\frac{a^{2}}{4}+\frac{a^{2} \log a}{2}-\frac{a \log a}{2}+\frac{\log a}{12}+\frac{1}{12} \\
& -\sum_{k=1}^{\infty} \frac{B_{2 k+2} a^{-2 k}}{(2 k+2)(2 k+1) 2 k},
\end{aligned}
$$

$$
\begin{aligned}
\zeta_{R}^{\prime}(-2, a) \sim & -\frac{a^{3}}{9}+\frac{a^{3} \log a}{3}-\frac{a^{2} \log a}{2}+\frac{a}{12}+\frac{a \log a}{6} \\
& +\sum_{k=1}^{\infty} \frac{2 B_{2 k+2} a^{-(2 k-1)}}{(2 k+2)(2 k+1) 2 k(2 k-1)},
\end{aligned}
$$

where $B_{k}$ are the Bernoulli numbers. 


\section{APPENDIX B: DERIVATION OF THE HIGH-l EXPANSION OF $f_{l}$}

This section is devoted to the derivation of $\ln f_{l}^{a}$ in (56) from the high- $l$ expansion of $\ln f_{l}$ in (51) up to $\mathcal{O}\left(\nu^{-4}\right)$, while keeping $\rho \rightarrow \infty$. For this purpose, let us first plug $V^{\prime \prime}$ from (55) into (51) and separate the integrals in three parts: the terms proportional to the delta function, to the Heaviside unit step function and the cross terms.

Delta function terms come purely from the discontinuity of the first derivative of the potential $\bar{\varphi}\left(R_{T}\right)=0$. One can compute the integrals exactly and perform the high- $l$ expansion from (A1). This gives the terms proportional to $\mu_{V} R_{T}$ in (56), one for each insertion of $V^{\prime \prime}$. For instance, the last three terms of (51), which are of third order in $V^{\prime \prime}$ are

$$
\begin{aligned}
\ln f_{l}^{a} & \supset-\frac{1}{3}\left(\mu_{V} R_{T} I_{\nu}(\sqrt{\gamma} \rho) K_{\nu}(\sqrt{\gamma} \rho)\right)^{3} \\
& \sim-\frac{1}{3}\left(\frac{t}{2 \nu} \mu_{V} R_{T}\right)^{3}
\end{aligned}
$$

where we kept all the terms up to $\mathcal{O}\left(\nu^{-4}\right)$.

Heaviside terms belong to the continuous part of $V^{\prime \prime}$. They were first computed by $[65,84]$ and contribute to the first term of (56). Let us proceed to compute each term of (51) by neglecting the delta terms.

The leading order terms in $V^{\prime \prime}$ can be computed simply by using the Bessel expansions in (A1). The second order terms in $V^{\prime \prime}$ can first be simplified by

$$
\int_{0}^{\infty} \mathrm{d} \rho_{1} \int_{\rho_{1}}^{\infty} \mathrm{d} \rho=\int_{0}^{\infty} \mathrm{d} \rho \int_{0}^{\rho} \mathrm{d} \rho_{1}
$$

since $V^{\prime \prime}$ is continuous, as shown in the Appendix E of $[100,101]$. At $\mathcal{O}\left(\nu^{-4}\right)$, this leads to

$$
\begin{aligned}
\ln f_{l}^{a} \supset & \sum_{s} \int_{0}^{\infty} \mathrm{d} \rho \rho \\
& \times \int_{0}^{\rho} \mathrm{d} \rho_{1} \rho_{1} K_{\nu}^{2}(\sqrt{\gamma} \rho) V_{s}^{\prime \prime}(\rho) V_{s}^{\prime \prime}\left(\rho_{1}\right) I_{\nu}^{2}\left(\sqrt{\gamma} \rho_{1}\right) \\
\sim & \frac{t^{3}}{8 \nu^{3}} \sum_{s} \int_{0}^{\infty} \mathrm{d} \rho \rho^{3} V_{s}^{\prime \prime 2} H\left((-1)^{s}\left(\rho-R_{T}\right)\right),
\end{aligned}
$$

where we used the exponential behavior of $I_{\nu}$ and $K_{\nu}$ in (A2) and the saddle-point approximation (A3) in the last step. Finally, the third order terms go as $\mathcal{O}\left(\nu^{-4}\right)$ and do not contribute to $f_{l}^{a}$ since each pair of Bessel functions (A2) as well as the saddle-point approximation (A1) come with a factor of $1 / \nu$.

Cross terms require a careful treatment in the integration of the delta function since it brings a Heaviside that affects the limits of integration of the second integration. Then we perform the asymptotic expansion of the Bessel functions and the saddle-point approximation as in the previous calculations with (A2) and (A3). These correspond to the last two terms of (56). For example, the last term of (56) is given by

$$
\begin{aligned}
\ln f_{l}^{a} \supset & \sum_{s} \int_{0}^{\infty} \mathrm{d} \rho \rho K_{\nu}^{2}(\sqrt{\gamma} \rho) V_{s}^{\prime \prime}(\rho) \\
& \times \int_{0}^{\rho} d \rho_{1} \rho_{1} \mu_{V} \delta\left(\rho-R_{T}\right) I_{\nu}^{2}\left(\sqrt{\gamma} \rho_{1}\right) \\
= & \mu_{V} R_{T} I_{\nu}^{2}\left(\sqrt{\gamma} R_{T}\right) \sum_{s} \int_{R_{T}}^{\infty} \mathrm{d} \rho \rho K_{\nu}^{2}(\sqrt{\gamma} \rho) V_{s}^{\prime \prime}(\rho) \\
& \times H\left(\rho-R_{T}\right) \\
\sim & \frac{1}{\mu_{V}^{2}}\left(\frac{t}{2 \nu} \mu_{V} R_{T}\right)^{3} V_{2}^{\prime \prime}\left(R_{T}\right),
\end{aligned}
$$

where in the second line, the integration limits have changed due to the previous integration of the delta function, which picks $V_{2}^{\prime \prime}$. ${ }^{8}$ Then we used the saddle point approximation that evaluates the potential at $R_{T}$ and provides the last line.

The next to last term of (56) can be computed completely analogously, while the remaining terms in (51) cancel among themselves or go as $\mathcal{O}\left(1 / \nu^{4}\right)$. After collecting all the results, we are left with the final expression given in (56).

\footnotetext{
${ }^{8}$ This actually depends on our convention of the Heaviside. We have chosen that $H(x)=0$ when $x<0$ but equivalently, we could have used $H(x)=1 / 2$ when $x=0$ and, after adding up all the cross terms in $\ln f_{l}^{a}$, we recover the same results.
}

[1] J. Langer, Theory of the condensation point, Ann. Phys. (N.Y.) 41, 108 (1967); 281, 941 (2000).

[2] I. Kobzarev, L. Okun, and M. Voloshin, Bubbles in metastable vacuum, Sov. J. Nucl. Phys. 20, 644 (1975).

[3] S. R. Coleman, The fate of the false vacuum. 1. Semiclassical theory, Phys. Rev. D 15, 2929 (1977).
[4] A. D. Linde, Fate of the false vacuum at finite temperature: Theory and applications, Phys. Lett. 100B, 37 (1981); Decay of the false vacuum at finite temperature, Nucl. Phys. B216, 421 (1983); Erratum, Nucl. Phys. B223, 544 (1983).

[5] A. I. Bochkarev, S. V. Kuzmin, and M. E. Shaposhnikov, Electroweak baryogenesis and the Higgs boson mass problem, Phys. Lett. B 244, 275 (1990). 
[6] A. G. Cohen, D. B. Kaplan, and A. E. Nelson, Weak scale baryogenesis, Phys. Lett. B 245, 561 (1990); Baryogenesis at the weak phase transition, Nucl. Phys. B349, 727 (1991).

[7] N. Turok and J. Zadrozny, Electroweak baryogenesis in the two doublet model, Nucl. Phys. B358, 471 (1991).

[8] Dynamical Generation of Baryons at the Electroweak Transition, Phys. Rev. Lett. 65, 2331 (1990).

[9] E. Witten, Cosmic separation of phases, Phys. Rev. D 30, 272 (1984).

[10] C. J. Hogan, Gravitational radiation from cosmological phase transitions, Mon. Not. R. Astron. Soc. 218, 629 (1986).

[11] A. Kosowsky, M. S. Turner, and R. Watkins, Gravitational Waves from First Order Cosmological Phase Transitions, Phys. Rev. Lett. 69, 2026 (1992); Gravitational radiation from colliding vacuum bubbles, Phys. Rev. D 45, 4514 (1992).

[12] C. Grojean and G. Servant, Gravitational waves from phase transitions at the electroweak scale and beyond, Phys. Rev. D 75, 043507 (2007).

[13] M. Hindmarsh, S. J. Huber, K. Rummukainen, and D. J. Weir, Gravitational Waves from the Sound of a First Order Phase Transition, Phys. Rev. Lett. 112, 041301 (2014); Numerical simulations of acoustically generated gravitational waves at a first order phase transition, Phys. Rev. D 92, 123009 (2015); Shape of the acoustic gravitational wave power spectrum from a first order phase transition, Phys. Rev. D 96, 103520 (2017).

[14] D. Cutting, M. Hindmarsh, and D. J. Weir, Gravitational waves from vacuum first-order phase transitions: From the envelope to the lattice, Phys. Rev. D 97, 123513 (2018); Vorticity, Kinetic Energy, and Suppressed Gravitational wave Production in Strong First Order Phase Transitions, Phys. Rev. Lett. 125, 021302 (2020).

[15] T. Vachaspati, Magnetic fields from cosmological phase transitions, Phys. Lett. B 265, 258 (1991).

[16] G. Sigl, A. V. Olinto, and K. Jedamzik, Primordial magnetic fields from cosmological first order phase transitions, Phys. Rev. D 55, 4582 (1997).

[17] A. De Simone, G. Nardini, M. Quiros, and A. Riotto, Magnetic fields at first order phase transition: A threat to electroweak baryogenesis, J. Cosmol. Astropart. Phys. 10 (2011) 030.

[18] A. G. Tevzadze, L. Kisslinger, A. Brandenburg, and T. Kahniashvili, Magnetic fields from QCD phase transitions, Astrophys. J. 759, 54 (2012).

[19] J. Ellis, M. Fairbairn, M. Lewicki, V. Vaskonen, and A. Wickens, Intergalactic magnetic fields from first-order phase transitions, J. Cosmol. Astropart. Phys. 09 (2019) 019.

[20] J. Aasi et al. (LIGO Scientific Collaboration), Advanced LIGO, Classical Quant. Grav. 32, 115012 (2015).

[21] F. Acernese et al. (Virgo Collaboration), Advanced Virgo: A second-generation interferometric gravitational wave detector, Classical Quant. Grav. 32, 024001 (2015).

[22] P. Amaro-Seoane et al. (LISA Collaboration), Laser interferometer space antenna, arXiv:1702.00786.

[23] C. Caprini et al., Science with the space-based interferometer eLISA. II: Gravitational waves from cosmological phase transitions, J. Cosmol. Astropart. Phys. 04 (2016) 001; Erratum, J. Cosmol. Astropart. Phys. 03 (2020) 024.

[24] S. Kawamura et al., The Japanese space gravitational wave antenna: DECIGO, Classical Quant. Grav. 28, 094011 (2011).

[25] J. Crowder and N. J. Cornish, Beyond LISA: Exploring future gravitational wave missions, Phys. Rev. D 72, 083005 (2005).

[26] V. Corbin and N. J. Cornish, Detecting the cosmic gravitational wave background with the big bang observer, Classical Quant. Grav. 23, 2435 (2006).

[27] S. Weinberg, Mass of the Higgs Boson, Phys. Rev. Lett. 36, 294 (1976).

[28] A. D. Linde, Dynamical symmetry restoration and constraints on masses and coupling constants in gauge theories, Pis'ma Zh. Eksp. Teor. Fiz. 23, 73 (1976), http://www.jetpletters.ac.ru/ps/1795/article_27429.shtml [JETP Lett. 23, 64 (1976)].

[29] P. H. Frampton, Consequences of vacuum instability in quantum field theory, Phys. Rev. D 15, 2922 (1977); Vacuum Instability and Higgs Scalar Mass, Phys. Rev. Lett. 37, 1378 (1976).

[30] G. Isidori, G. Ridolfi, and A. Strumia, On the metastability of the standard model vacuum, Nucl. Phys. B609, 387 (2001).

[31] A. Andreassen, W. Frost, and M. D. Schwartz, Scale invariant instantons and the complete lifetime of the standard model, Phys. Rev. D 97, 056006 (2018).

[32] R. P. Feynman and A. R. Hibbs, Quantum Mechanics and Path Integrals (McGraw-Hill, NewYork, 1965), Emended by (Dover, Mineola, New York, 2010).

[33] H. Kleinert, Path Integrals in Quantum Mechanics, Statistics, Polymer Physics, and Financial Markets, 5th ed. (World Scientific, Singapore, 2009).

[34] G. Derrick, Comments on nonlinear wave equations as models for elementary particles, J. Math. Phys. (N.Y.) 5, 1252 (1964).

[35] S. R. Coleman, V. Glaser, and A. Martin, Action minima among solutions to a class of Euclidean scalar field equations, Commun. Math. Phys. 58, 211 (1978).

[36] S. Fubini, A new approach to conformal invariant field theories, Nuovo Cimento A 34, 521 (1976).

[37] L. Lipatov, Divergence of the perturbation theory series and the quasiclassical theory, Sov. Phys. JETP 45, 216 (1977), https://inspirehep.net/literature/111423.

[38] F. Loran, Fubini vacua as a classical de Sitter vacua, Mod. Phys. Lett. A 22, 2217 (2007).

[39] M. J. Duncan and L. G. Jensen, Exact tunneling solutions in scalar field theory, Phys. Lett. B 291, 109 (1992).

[40] K. Dutta, C. Hector, T. Konstandin, P. M. Vaudrevange, and A. Westphal, Validity of the kink approximation to the tunneling action, Phys. Rev. D 86, 123517 (2012).

[41] V. Guada, A. Maiezza, and M. Nemevšek, Multifield polygonal bounces, Phys. Rev. D 99, 056020 (2019).

[42] A. Ferraz de Camargo, R. C. Shellard, and G. C. Marques, Vacuum decay in a soluble model, Phys. Rev. D 29, 1147 (1984).

[43] A. Aravind, B. S. DiNunno, D. Lorshbough, and S. Paban, Analyzing multifield tunneling with exact bounce solutions, Phys. Rev. D 91, 025026 (2015). 
[44] T. C. Shen, Bubbles without cores, Phys. Rev. D 37, 3537 (1988).

[45] K. M. Lee and E. J. Weinberg, Tunneling without barriers, Nucl. Phys. B267, 181 (1986).

[46] K. Dutta, C. Hector, P. M. Vaudrevange, and A. Westphal, More exact tunneling solutions in scalar field theory, Phys. Lett. B 708, 309 (2012).

[47] C. L. Wainwright, CosmoTransitions: Computing cosmological phase transition temperatures and bubble profiles with multiple fields, Comput. Phys. Commun. 183, 2006 (2012).

[48] A. Masoumi, K. D. Olum, and B. Shlaer, Efficient numerical solution to vacuum decay with many fields, J. Cosmol. Astropart. Phys. 01 (2017) 051.

[49] P. Athron, C. Balázs, M. Bardsley, A. Fowlie, D. Harries, and G. White, BubbleProfiler: Finding the field profile and action for cosmological phase transitions, Comput. Phys. Commun. 244, 448 (2019).

[50] R. Sato, SimpleBounce: A simple package for the false vacuum decay, Comput. Phys. Commun. 258, 107566 (2021).

[51] V. Guada, M. Nemevšek, and M. Pintar, FindBounce: Package for multi-field bounce actions, Comput. Phys. Commun. 256, 107480 (2020).

[52] K. A. Intriligator, N. Seiberg, and D. Shih, Dynamical SUSY breaking in meta-stable vacua, J. High Energy Phys. 04 (2006) 021.

[53] M. Carena, S. Gori, I. Low, N. R. Shah, and C.E. M. Wagner, Vacuum stability and Higgs diphoton decays in the MSSM, J. High Energy Phys. 02 (2013) 114.

[54] G. Pastras, Exact tunneling solutions in Minkowski spacetime and a candidate for dark energy, J. High Energy Phys. 08 (2013) 075.

[55] J. Jaeckel, V. V. Khoze, and M. Spannowsky, Hearing the signal of dark sectors with gravitational wave detectors, Phys. Rev. D 94, 103519 (2016).

[56] Z. Zhou, J. Yan, A. Addazi, Y. F. Cai, A. Marciano, and R. Pasechnik, Probing new physics with multi-vacua quantum tunnelings beyond standard model through gravitational waves, arXiv:2003.13244.

[57] K. Dutta, P. M. Vaudrevange, and A. Westphal, An exact tunneling solution in a simple realistic landscape, Classical Quant. Grav. 29, 065011 (2012).

[58] K. Kamada, Inflationary cosmology and the standard model Higgs with a small Hubble induced mass, Phys. Lett. B 742, 126 (2015).

[59] A. A. Andrianov, F. Cannata, and A. Y. Kamenshchik, General solution of scalar field cosmology with a (piecewise) exponential potential, J. Cosmol. Astropart. Phys. 10 (2011) 004.

[60] M. Gleiser, Metastability in the early universe. 1, Phys. Rev. D 42, 3350 (1990).

[61] M. Gleiser and A. F. Heckler, Nonperturbative Effects on Nucleation, Phys. Rev. Lett. 76, 180 (1996).

[62] M. Gleiser, B. Rogers, and J. Thorarinson, Bubbling the false vacuum away, Phys. Rev. D 77, 023513 (2008).

[63] C. G. Callan, Jr. and S. R. Coleman, The fate of the false vacuum. 2. First quantum corrections, Phys. Rev. D 16, 1762 (1977).
[64] J. Baacke and G. Lavrelashvili, One loop corrections to the metastable vacuum decay, Phys. Rev. D 69, 025009 (2004).

[65] G. V. Dunne and H. Min, Beyond the thin-wall approximation: Precise numerical computation of prefactors in false vacuum decay, Phys. Rev. D 72, 125004 (2005).

[66] J. Hur and H. Min, A fast way to compute functional determinants of radially symmetric partial differential operators in general dimensions, Phys. Rev. D 77, 125033 (2008).

[67] A. Strumia and N. Tetradis, A consistent calculation of bubble nucleation rates, Nucl. Phys. B542, 719 (1999).

[68] G. Munster and S. Rotsch, Analytical calculation of the nucleation rate for first order phase transitions beyond the thin wall approximation, Eur. Phys. J. C 12, 161 (2000).

[69] K. Blum, M. Honda, R. Sato, M. Takimoto, and K. Tobioka, $\mathrm{O}(N)$ invariance of the multi-field bounce, J. High Energy Phys. 05 (2017) 109; Erratum, 06 (2017) 60.

[70] S. Chigusa, T. Moroi, and Y. Shoji, Precise calculation of the decay rate of false vacuum with multi-field bounce, J. High Energy Phys. 11 (2020) 006.

[71] R. V. Konoplich, Calculation of quantum corrections to nontrivial classical solutions by means of the Zeta function, Theor. Math. Phys. 73, 1286 (1987).

[72] A. W. Wipf, Tunnel determinants, Nucl. Phys. B269, 24 (1986).

[73] B. Garbrecht and P. Millington, Green's function method for handling radiative effects on false vacuum decay, Phys. Rev. D 91, 105021 (2015).

[74] W. Y. Ai, B. Garbrecht, and P. Millington, Radiative effects on false vacuum decay in Higgs-Yukawa theory, Phys. Rev. D 98, 076014 (2018).

[75] M. Endo, T. Moroi, M. M. Nojiri, and Y. Shoji, On the gauge invariance of the decay rate of false vacuum, Phys. Lett. B 771, 281 (2017).

[76] M. Endo, T. Moroi, M. M. Nojiri, and Y. Shoji, False vacuum decay in gauge theory, J. High Energy Phys. 11 (2017) 074.

[77] B. Garbrecht and P. Millington, Fluctuations about the Fubini-Lipatov instanton for false vacuum decay in classically scale invariant models, Phys. Rev. D 98, 016001 (2018).

[78] D. Croon, O. Gould, P. Schicho, T. V. I. Tenkanen, and G. White, Theoretical uncertainties for cosmological firstorder phase transitions, arXiv:2009.10080.

[79] I. Gelfand and A. Yaglom, Integration in functional spaces and it applications in quantum physics, J. Math. Phys. (N.Y.) 1, 48 (1960).

[80] S. R. Coleman, Quantum tunneling and negative eigenvalues, Nucl. Phys. B298, 178 (1988).

[81] A. Kusenko, Tunneling in quantum field theory with spontaneous symmetry breaking, Phys. Lett. B 358, 47 (1995).

[82] A. Kusenko, K. M. Lee, and E. J. Weinberg, Vacuum decay and internal symmetries, Phys. Rev. D 55, 4903 (1997).

[83] K. Kirsten and P. Loya, Computation of determinants using contour integrals, Am. J. Phys. 76, 60 (2008).

[84] G. V. Dunne and K. Kirsten, Functional determinants for radial operators, J. Phys. A 39, 11915 (2006).

[85] C. L. Hammer, J.E. Shrauner, and B. DeFacio, Alternate derivation of vacuum tunneling, Phys. Rev. D 19, 667 (1979). 
[86] S. R. Coleman, The uses of instantons, Subnucl. Ser. 15, 805 (1979).

[87] S. R. Coleman, Aspects of Symmetry: Selected Erice Lectures (Cambridge University Press, Cambridge, England, 1985).

[88] E. J. Weinberg, Classical Solutions in Quantum Field Theory, Cambridge Monographs on Mathematical Physics (Cambridge University Press, Cambridge, England, 2012).

[89] S. Weinberg, The Quantum Theory of Fields. Vol. 2: Modern Applications (Cambridge University Press, Cambridge, England, 2013).

[90] A. Andreassen, D. Farhi, W. Frost, and M. D. Schwartz, Direct Approach to Quantum Tunneling, Phys. Rev. Lett. 117, 231601 (2016).

[91] A. Andreassen, D. Farhi, W. Frost, and M. D. Schwartz, Precision decay rate calculations in quantum field theory, Phys. Rev. D 95, 085011 (2017).

[92] J.-L. Gervais and B. Sakita, Extended particles in quantum field theories, Phys. Rev. D 11, 2943 (1975).

[93] J.-L. Gervais, A. Jevicki, and B. Sakita, Perturbation expansion around extended particle states in quantum field theory. 1, Phys. Rev. D 12, 1038 (1975).

[94] C. G. Callan, Jr. and D. J. Gross, Quantum perturbation theory of solitons, Nucl. Phys. B93, 29 (1975).

[95] A. Jevicki, Treatment of zero frequency modes in perturbation expansion about classical field configurations, Nucl. Phys. B117, 365 (1976).

[96] S. Oda, Y. Shoji, and D. S. Takahashi, High scale validity of the DFSZ axion model with precision, J. High Energy Phys. 03 (2020) 011.

[97] S. Minakshisundaram and A. Pleijel, Some properties of the eigenfunctions of the Laplace operator on Riemannian manifolds, Can. J. Math. 1, 242 (1949).

[98] S. W. Hawking, Zeta function regularization of path integrals in curved space-time, Commun. Math. Phys. 55, 133 (1977).

[99] E. Elizalde, S. D. Odintsov, A. Romeo, A. A. Bytsenko, and S. Zerbini, Zeta Regularization Techniques with Applications (World Scientific, Singapore, 1994).

[100] K. Kirsten, Spectral functions in mathematics and physics, AIP Conf. Proc. 484, 106 (1999).
[101] K. Kirsten, Spectral Functions in Mathematics and Physics (Chapman \& Hall/CRC Press, Boca Raton, FL, 2002); AIP Conf. Proc. 484, 106 (1999).

[102] G. V. Dunne, Functional determinants in quantum field theory, J. Phys. A 41, 304006 (2008); Lecture notes on functional determinants in quantum field theory at the 14th WE Heraeus Saalburg summer school in Wolfersdorf.

[103] K. Kirsten and A. J. McKane, Functional determinants by contour integration methods, Ann. Phys. (Amsterdam) 308, 502 (2003).

[104] K. Kirsten and A. J. McKane, Functional determinants in the presence of zero modes, arXiv:hep-th/0507005.

[105] K. Kirsten and A. J. McKane, Functional determinants for general Sturm-Liouville problems, J. Phys. A 37, 4649 (2004).

[106] H. Weyl, Das asymptotische Verteilungsgesetz der Eigenwerte linearer partieller Differentialgleichungen (mit einer Anwendung auf die Theorie der Hohlraumstrahlung), Math. Ann. 71, 441 (1912).

[107] See Supplemental Material at http://link.aps.org/ supplemental/10.1103/PhysRevD.102.125017, for the Mathematica notebook with the entire calculation of the false vacuum decay rate for the quartic-quartic potential and its complexified version. This includes the bounce action and the one loop finite and renormalized parts.

[108] W. Y. Ai, J. S. Cruz, B. Garbrecht, and C. Tamarit, Gradient effects on false vacuum decay in gauge theory, Phys. Rev. D 102, 085001 (2020).

[109] T. Konstandin and S. J. Huber, Numerical approach to multi dimensional phase transitions, J. Cosmol. Astropart. Phys. 06 (2006) 021.

[110] V. Guada and M. Nemevšek (to be published).

[111] M. Abramowitz and I. A. Stegun, Handbook of Mathematical Functions with Formulas, Graphs, and Mathematical Tables, Appl. Math. Ser. Vol. 55 (Dover Publications, New York, 1974).

[112] E. Elizalde, An asymptotic expansion for the first derivative of the generalized Riemann zeta function, Math. Comput. 47, 347 (1986). 